\title{
OBSERVATION AND CHARACTERIZATION OF A COSMIC MUON NEUTRINO FLUX FROM THE NORTHERN HEMISPHERE USING SIX YEARS OF ICECUBE DATA
}

M. G. Aartsen ${ }^{1}$, K. Abraham ${ }^{2}$, M. Ackermann ${ }^{3}$, J. Adams ${ }^{4}$, J. A. Agullar ${ }^{5}$, M. Ahlers ${ }^{6}$, M. Ahrens ${ }^{7}$, D. Altmann ${ }^{8}$,

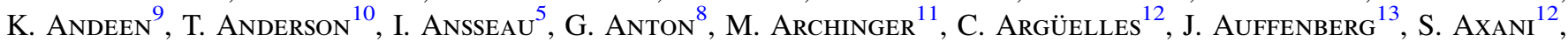
X. Bai ${ }^{14}$, S. W. Barwick ${ }^{15}$, V. Baum ${ }^{11}$, R. BaY ${ }^{16}$, J. J. BeattY ${ }^{17,18}$, J. Becker Tuus ${ }^{19}$, K.-H. Becker ${ }^{20}$, S. BenZvi ${ }^{21}$, P. Berghaus ${ }^{22}$, D. Berley ${ }^{23}$, E. Bernardini ${ }^{3}$, A. Bernhard ${ }^{2}$, D. Z. Besson ${ }^{24}$, G. Binder ${ }^{16,25}$, D. Bindig ${ }^{20}$, M. BissoK ${ }^{13}$, E. Blaufuss ${ }^{23}$, S. Blot ${ }^{3}$, C. Bohm ${ }^{7}$, M. Börner ${ }^{26}$, F. Bos $^{19}$, D. Bose ${ }^{27}$, S. Böser ${ }^{11}$, O. Botner ${ }^{28}$, J. Braun ${ }^{6}$, L. BraYeur ${ }^{29}$, H.-P. Bretz ${ }^{3}$, A. Burgman ${ }^{28}$, T. Carver ${ }^{30}$, M. Casier ${ }^{29}$, E. Cheung ${ }^{23}$, D. Chirkin ${ }^{6}$, A. Christov ${ }^{30}$, K. Clark ${ }^{31}$, L. Classen $^{32}$, S. Coenders' ${ }^{2}$, G. H. Collin ${ }^{12}$, J. M. Conrad ${ }^{12}$, D. F. Cowen ${ }^{10,33}$, R. Cross ${ }^{21}$, M. DAY ${ }^{6}$, J. P. A. M. DE ANDrÉ ${ }^{34}$,

C. De Clerce ${ }^{29}$, E. del Pino Rosendo ${ }^{11}$, H. Dembinski ${ }^{35}$, S. De Ridder ${ }^{36}$, P. Desiati ${ }^{6}$, K. D. De Vries ${ }^{29}$, G. De Wasseige ${ }^{29}$, M. De With ${ }^{37}$, T. DeYoung ${ }^{34}$, J. C. Díaz-VÉlez ${ }^{6}$, V. Di Lorenzo ${ }^{11}$, H. Dujmovic $^{27}$, J. P. Dumm ${ }^{7}$, M. DunKman ${ }^{10}$,

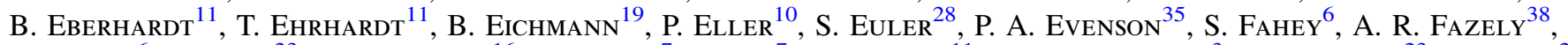
J. Feintzeig ${ }^{6}$, J. Felde ${ }^{23}$, K. Filimonov ${ }^{16}$, C. Finley ${ }^{7}$, S. Flis ${ }^{7}$, C.-C. Fösig ${ }^{11}$, A. Franckowiak ${ }^{3}$, E. Friedman ${ }^{23}$, T. FuChs ${ }^{26}$, T. K. Gaisser ${ }^{35}$, J. Gallagher ${ }^{39}$, L. Gerhardt ${ }^{16,25}$, K. Ghorbani ${ }^{6}$, W. Giang ${ }^{40}$, L. Gladstone ${ }^{6}$, M. Glagla ${ }^{13}$, T. Glüsenkamp ${ }^{3}$, A. Goldschmidt ${ }^{25}$, G. Golup ${ }^{29}$, J. G. GonZalez ${ }^{35}$, D. Grant ${ }^{40}$, Z. Griffith ${ }^{6}$, C. HaAcK $^{13}$, A. Haj Ismail $^{36}$,

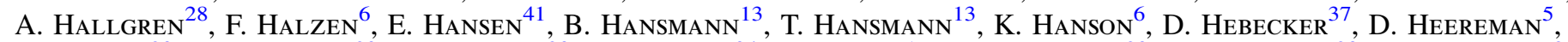

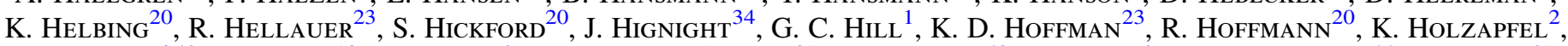

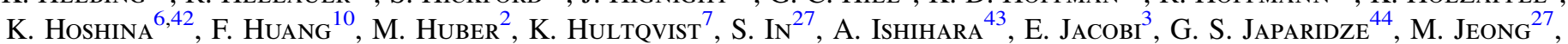

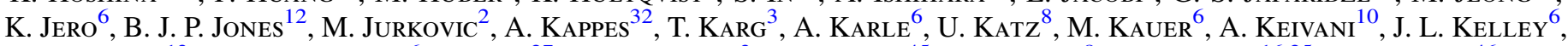

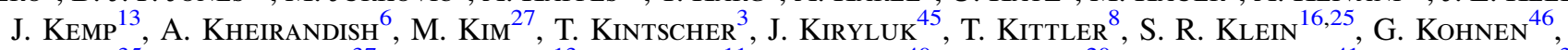

R. Koirala ${ }^{35}$, H. Kolanoski ${ }^{37}$, R. Konietz ${ }^{13}$, L. KöPKe ${ }^{11}$, C. Kopper ${ }^{40}$, S. Kopper ${ }^{20}$, D. J. Koskinen ${ }^{41}$, M. KowalsKI ${ }^{3}, 37$, K. Krings 2 , M. Kroll ${ }^{19}$, G. KrÜCKL ${ }^{11}$, C. KrÜGer ${ }^{6}$, J. Kunnen ${ }^{29}$, S. Kunwar ${ }^{3}$, N. Kurahashi ${ }^{47}$, T. Kuwabara ${ }^{43}$, M. Labare $^{36}$, J. L. Lanfranchi ${ }^{10}$, M. J. Larson ${ }^{41}$, F. Lauber ${ }^{20}$, D. Lennarz ${ }^{34}$, M. LesiaK-BzdaK ${ }^{45}$, M. LeuermanN ${ }^{13}$,

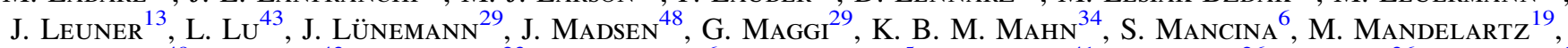
R. Maruyama ${ }^{49}$, K. Mase ${ }^{43}$, R. Maunu ${ }^{23}$, F. McNally ${ }^{6}$, K. Meagher ${ }^{5}$, M. Medici ${ }^{41}$, M. Meier ${ }^{26}$, A. Meli ${ }^{36}$, T. Menne ${ }^{26}$, G. Merino $^{6}$, T. Meures ${ }^{5}$, S. Miarecki ${ }^{16,25}$, L. MohrmanN ${ }^{3}$, T. Montaruli ${ }^{30}$, M. Moulai $^{12}$, R. Nahnhauer ${ }^{3}$, U. Naumann ${ }^{20}$, G. Neer ${ }^{34}$, H. Niederhausen ${ }^{45}$, S. C. Nowicki ${ }^{40}$, D. R. Nygren ${ }^{25}$, A. Obertacke Pollmann ${ }^{20}$, A. Olivas ${ }^{23}$,

A. O’Murchadha ${ }^{5}$, T. Palczewski ${ }^{50}$, H. Pandya ${ }^{35}$, D. V. Pankova ${ }^{10}$, P. Peiffer ${ }^{11}$, Ö. Penek ${ }^{13}$, J. A. Pepper ${ }^{50}$,

C. Pérez de los $\mathrm{Heros}^{28}$, D. Pieloth ${ }^{26}$, E. Pinat ${ }^{5}$, P. B. Price ${ }^{16}$, G. T. Przybylski $^{25}$, M. Quinnan ${ }^{10}$, C. RaAB $^{5}$, L. RäDel ${ }^{13}$, M. Rameez ${ }^{41}$, K. Rawlins ${ }^{51}$, R. Reimann ${ }^{13}$, B. Relethford ${ }^{47}$, M. Relich ${ }^{43}$, E. Resconi ${ }^{2}$, W. Rhode ${ }^{26}$, M. Richman ${ }^{47}$,

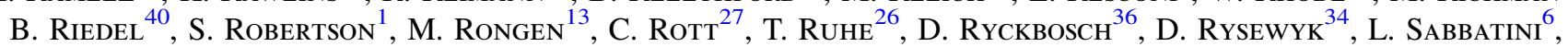
S. E. Sanchez Herrera ${ }^{40}$, A. Sandrock ${ }^{26}$, J. SAndroos ${ }^{11}$, S. Sarkar ${ }^{41,52}$, K. Satalecka ${ }^{3}$, M. Schimp ${ }^{13}$, P. Schlunder ${ }^{26}$, T. Schmidt ${ }^{23}$, S. Schoenen ${ }^{13}$, S. Schöneberg ${ }^{19}$, L. Schumacher ${ }^{13}$, D. Seckel ${ }^{35}$, S. Seunarine ${ }^{48}$, D. Soldin ${ }^{20}$, M. Song $^{23}$, G. M. SpiczaK ${ }^{48}$, C. Spiering ${ }^{3}$, M. Stahlberg ${ }^{13}$, T. Stanev ${ }^{35}$, A. StasiK ${ }^{3}$, A. Steuer ${ }^{11}$, T. Stezelberger ${ }^{25}$, R. G. Stokstad ${ }^{25}$, A. StößL ${ }^{3}$, R. Ström ${ }^{28}$, N. L. Strotjohann ${ }^{3}$, G. W. Sullivan ${ }^{23}$, M. Sutherland ${ }^{17}$, H. TaAvola ${ }^{28}$, I. Taboada ${ }^{53}$, J. TataR $^{16,25}$, F. Tenholit ${ }^{19}$, S. Ter-Antonyan ${ }^{38}$, A. Terliuk ${ }^{3}$, G. Tešić ${ }^{10}$, S. Tilav ${ }^{35}$, P. A. Toale ${ }^{50}$, M. N. Tobin ${ }^{6}$, S. Toscano $^{29}$, D. Tosi $^{6}$,

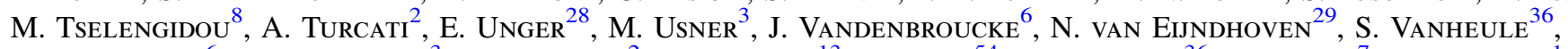
M. van Rossem ${ }^{6}$, J. van Santen $^{3}$, J. Veenkamp ${ }^{2}$, M. Vehring $^{13}$, M. Voge $^{54}$, M. VRaeghe $^{36}$, C. Walck ${ }^{7}$, A. Wallace ${ }^{1}$,

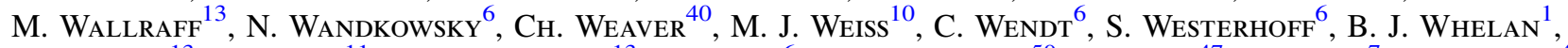
S. WickmanN ${ }^{13}$, K. Wiebe $^{11}$, C. H. Wiebusch ${ }^{13}$, L. WilleE ${ }^{6}$, D. R. Williams ${ }^{50}$, L. Wills ${ }^{47}$, M. Wolf ${ }^{7}$, T. R. Wood ${ }^{40}$,

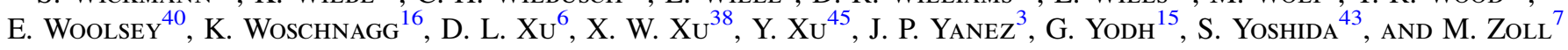

(ICecube Collaboration)

${ }^{1}$ Department of Physics, University of Adelaide, Adelaide, 5005, Australia

${ }^{2}$ Physik-department, Technische Universität München, D-85748 Garching, Germany ${ }^{3}$ DESY, D-15735 Zeuthen, Germany

${ }^{4}$ Dept. of Physics and Astronomy, University of Canterbury, Private Bag 4800, Christchurch, New Zealand ${ }^{5}$ Université Libre de Bruxelles, Science Faculty CP230, B-1050 Brussels, Belgium

${ }^{6}$ Dept. of Physics and Wisconsin IceCube Particle Astrophysics Center, University of Wisconsin, Madison, WI 53706, USA 7 Oskar Klein Centre And Dept. Of Physics, Stockholm University, SE-10691 Stockholm, Sweden

${ }^{8}$ Erlangen Centre for Astroparticle Physics, Friedrich-Alexander-Universität Erlangen-Nürnberg, D-91058 Erlangen, Germany ${ }^{9}$ Department of Physics, Marquette University, Milwaukee, WI, 53201, USA

${ }^{10}$ Dept. of Physics, Pennsylvania State University, University Park, PA 16802, USA

${ }^{11}$ Institute of Physics, University of Mainz, Staudinger Weg 7, D-55099 Mainz, Germany

12 Dept. of Physics, Massachusetts Institute of Technology, Cambridge, MA 02139, USA

${ }^{13}$ III. Physikalisches Institut, RWTH Aachen University, D-52056 Aachen, Germany

${ }^{14}$ Physics Department, South Dakota School of Mines and Technology, Rapid City, SD 57701, USA

${ }^{15}$ Dept. of Physics and Astronomy, University of California, Irvine, CA 92697, USA

${ }^{17}$ Dept. of Physics and Center for Cosmology and Astro-Particle Physics, Ohio State University, Columbus, OH 43210, USA 


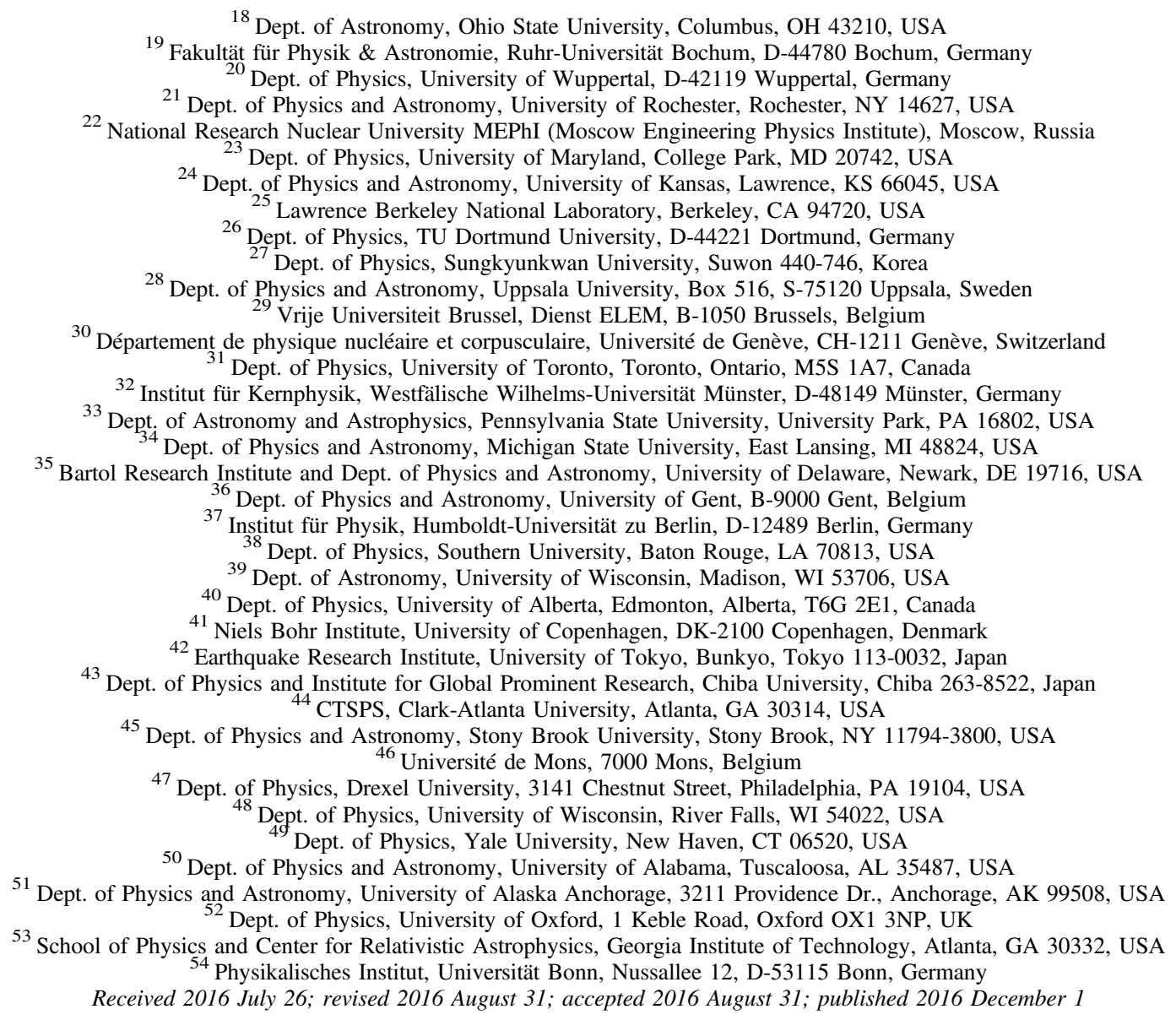

${ }^{18}$ Dept. of Astronomy, Ohio State University, Columbus, OH 43210, USA

${ }^{19}$ Fakultät für Physik \& Astronomie, Ruhr-Universität Bochum, D-44780 Bochum, Germany

${ }^{20}$ Dept. of Physics, University of Wuppertal, D-42119 Wuppertal, Germany

${ }^{21}$ Dept. of Physics and Astronomy, University of Rochester, Rochester, NY 14627, USA

${ }^{22}$ National Research Nuclear University MEPhI (Moscow Engineering Physics Institute), Moscow, Russia

${ }^{23}$ Dept. of Physics, University of Maryland, College Park, MD 20742, USA

${ }^{24}$ Dept. of Physics and Astronomy, University of Kansas, Lawrence, KS 66045, USA

${ }^{25}$ Lawrence Berkeley National Laboratory, Berkeley, CA 94720, USA

${ }^{26}$ Dept. of Physics, TU Dortmund University, D-44221 Dortmund, Germany

27 Dept. of Physics, Sungkyunkwan University, Suwon 440-746, Korea

${ }^{28}$ Dept. of Physics and Astronomy, Uppsala University, Box 516, S-75120 Uppsala, Sweden

${ }^{29}$ Vrije Universiteit Brussel, Dienst ELEM, B-1050 Brussels, Belgium

${ }^{30}$ Département de physique nucléaire et corpusculaire, Université de Genève, CH-1211 Genève, Switzerland

${ }^{31}$ Dept. of Physics, University of Toronto, Toronto, Ontario, M5S 1A7, Canada

${ }^{32}$ Institut für Kernphysik, Westfälische Wilhelms-Universität Münster, D-48149 Münster, Germany

${ }^{33}$ Dept. of Astronomy and Astrophysics, Pennsylvania State University, University Park, PA 16802, USA

34 Dept. of Physics and Astronomy, Michigan State University, East Lansing, MI 48824, USA

${ }^{35}$ Bartol Research Institute and Dept. of Physics and Astronomy, University of Delaware, Newark, DE 19716, USA

${ }_{37}^{36}$ Dept. of Physics and Astronomy, University of Gent, B-9000 Gent, Belgium

${ }^{37}$ Institut für Physik, Humboldt-Universität zu Berlin, D-12489 Berlin, Germany

${ }^{38}$ Dept. of Physics, Southern University, Baton Rouge, LA 70813, USA

${ }^{39}$ Dept. of Astronomy, University of Wisconsin, Madison, WI 53706, USA

${ }^{40}$ Dept. of Physics, University of Alberta, Edmonton, Alberta, T6G 2E1, Canada

${ }^{41}$ Niels Bohr Institute, University of Copenhagen, DK-2100 Copenhagen, Denmark

${ }^{42}$ Earthquake Research Institute, University of Tokyo, Bunkyo, Tokyo 113-0032, Japan

${ }^{43}$ Dept. of Physics and Institute for Global Prominent Research, Chiba University, Chiba 263-8522, Japan

${ }^{44}$ CTSPS, Clark-Atlanta University, Atlanta, GA 30314, USA

${ }^{45}$ Dept. of Physics and Astronomy, Stony Brook University, Stony Brook, NY 11794-3800, USA ${ }^{46}$ Université de Mons, 7000 Mons, Belgium

${ }^{47}$ Dept. of Physics, Drexel University, 3141 Chestnut Street, Philadelphia, PA 19104, USA

${ }^{48}$ Dept. of Physics, University of Wisconsin, River Falls, WI 54022, USA

${ }^{50}$ Dept. of Physics, Yale University, New Haven, CT 06520, USA
Dept. of Physics and Astronomy, University of Alabama, Tuscaloosa, AL 35487, USA

${ }^{51}$ Dept. of Physics and Astronomy, University of Alaska Anchorage, 3211 Providence Dr., Anchorage, AK 99508, USA

${ }^{52}$ Dept. of Physics, University of Oxford, 1 Keble Road, Oxford OX1 3NP, UK

${ }^{53}$ School of Physics and Center for Relativistic Astrophysics, Georgia Institute of Technology, Atlanta, GA 30332, USA

${ }_{54}$ Physikalisches Institut, Universität Bonn, Nussallee 12, D-53115 Bonn, Germany

Received 2016 July 26; revised 2016 August 31; accepted 2016 August 31; published 2016 December 1

\begin{abstract}
The IceCube Collaboration has previously discovered a high-energy astrophysical neutrino flux using neutrino events with interaction vertices contained within the instrumented volume of the IceCube detector. We present a complementary measurement using charged current muon neutrino events where the interaction vertex can be outside this volume. As a consequence of the large muon range the effective area is significantly larger but the field of view is restricted to the Northern Hemisphere. IceCube data from 2009 through 2015 have been analyzed using a likelihood approach based on the reconstructed muon energy and zenith angle. At the highest neutrino energies between $194 \mathrm{TeV}$ and $7.8 \mathrm{PeV}$ a significant astrophysical contribution is observed, excluding a purely atmospheric origin of these events at 5.6 $\sigma$ significance. The data are well described by an isotropic, unbroken power-law flux with a normalization at $100 \mathrm{TeV}$ neutrino energy of $\left(0.90_{-0.27}^{+0.30}\right) \times 10^{-18} \mathrm{GeV}^{-1} \mathrm{~cm}^{-2} \mathrm{~s}^{-1} \mathrm{sr}^{-1}$ and a hard spectral index of $\gamma=2.13 \pm 0.13$. The observed spectrum is harder in comparison to previous IceCube analyses with lower energy thresholds which may indicate a break in the astrophysical neutrino spectrum of unknown origin. The highest-energy event observed has a reconstructed muon energy of $(4.5 \pm 1.2) \mathrm{PeV}$ which implies a probability of less than $0.005 \%$ for this event to be of atmospheric origin. Analyzing the arrival directions of all events with reconstructed muon energies above $200 \mathrm{TeV}$ no correlation with known $\gamma$-ray sources was found. Using the high statistics of atmospheric neutrinos we report the current best constraints on a prompt atmospheric muon neutrino flux originating from charmed meson decays which is below 1.06 in units of the flux normalization of the model in Enberg et al.
\end{abstract}

Key words: astroparticle physics - methods: data analysis - neutrinos

\section{INTRODUCTION}

The detection of high-energy cosmic neutrinos as cosmic messengers has been an important goal of astroparticle physics. Being stable, electrically neutral particles, high-energy neutrinos are able to propagate almost undisturbed through the universe from their production sites to Earth keeping their directional and energy information. Hence, they constitute excellent cosmic messenger particles, particularly at the highest energies. They arise from weak decays of hadrons, mostly pions and kaons, which are expected to be produced by hadronic interactions of cosmic rays in the surrounding matter of the cosmic-ray accelerator. Their observation will help to elucidate the unknown sources of high-energy cosmic rays (Gaisser et al. 1995; Learned \& Mannheim 2000; Becker 2008).

Already in the 1960s the observation of high-energy neutrinos had been discussed by Greisen (1960), Markov 
(1960), and Reines (1960), shortly after the discovery of the neutrino by Reines \& Cowan (1956). The proposed method was the detection of up-going muons as a signature of a charged-current (CC) muon neutrino interaction below the detector. Soon it was realized that the expected astrophysical fluxes are small and cubic-kilometer-sized detectors would be needed to accomplish the goal, see e.g., Roberts (1992). The construction of large Cherenkov detectors by instrumenting optically transparent natural media, i.e., deep oceans, lakes, and glaciers with photo-sensors (Belolaptikov et al. 1997; Andres et al. 2000; Ageron et al. 2011) proved to be a key concept. The largest instrument to date is the IceCube Neutrino Observatory at the geographic South Pole (Achterberg et al. 2006).

Main backgrounds to the search for astrophysical neutrinos are high-energy atmospheric neutrinos and muons produced by cosmic-ray interactions in the Earth's atmosphere.

In 2013, a diffuse all-flavor flux of high-energy astrophysical neutrinos was discovered (Aartsen et al. 2013a, 2014b). The analysis selected events due to high-energy neutrinos which interact within the detector by using its outer layers as a veto. This strategy enables a full-sky sensitivity for all neutrino flavors. The veto not only rejects atmospheric muons entering the detector from the outside extremely efficiently, but also atmospheric neutrinos from above the detector which are produced together with muons.

In this analysis we focus on up-going muons which arise from charged-current interactions of muon neutrinos both inside and outside the detector. By allowing neutrinos to interact outside the instrumented volume a larger effective area is achieved. However, at the same time it is necessary to restrict the analysis to the Northern Hemisphere where the Earth filters atmospheric muons efficiently. Furthermore, the analysis is mainly sensitive to a muon neutrino flux because of the large muon range. Nevertheless this strategy will impose further constraints on possible models (Cholis \& Hooper 2013; He et al. 2013; Kalashev et al. 2013; Laha et al. 2013; Razzaque 2013; Roulet et al. 2013; Stecker 2013; Anchordoqui et al. 2014; Gonzalez-Garcia et al. 2014; Murase et al. 2014; Tamborra et al. 2014; Bechtol et al. 2015; Senno et al. 2016) that have been proposed to explain the observed astrophysical neutrino flux.

This analysis is based on a high-purity and high-statistics selection of about 350,000 well-reconstructed up-going muon events from six years of IceCube operation, improving the statistics compared to previous analyses (Aartsen et al. 2014c, 2015c) by almost an order of magnitude.

Even when individual astrophysical neutrino sources cannot be identified because they are too weak, their cumulative flux can be measured as a diffuse flux. The signature of an astrophysical neutrino signal with respect to the background of atmospheric neutrinos is illustrated in Figure 1. Astrophysical neutrinos from cosmic accelerators are generically expected to have a hard energy spectrum as originally predicted by Fermi: $d N_{\nu} / d E \simeq \phi_{0} \cdot E^{-2}$. However, the spectral index depends in detail on the source properties and the acceleration mechanism (Bell 2013; Kashti \& Waxman 2005; Klein et al. 2013). Recent IceCube analyses (Kopper et al. 2015; Aartsen et al. 2015a, 2015b; Lesiak-Bzdak et al. 2015) yielded a softer spectrum with a spectral index between 2.5 and 2.7.

The energy spectrum of the atmospheric neutrino background is about one power steeper than the primary cosmic-ray spectrum $\left(d N_{\mathrm{CR}} / d E \propto E^{-2.7 . .3 .1}\right)$, with the exception of prompt neutrinos from heavy meson decays, which follow the primary spectrum more closely. The astrophysical signal appears as an excess above energies of about $100 \mathrm{TeV}$. As shown, the zenith distribution differs for signal and backgrounds which themselves depend on the energy. At the highest energies the Earth becomes increasingly opaque to neutrinos and the signal is dominated by events near the horizon.

The identification of an astrophysical signal is based on a two-dimensional likelihood fit in zenith and energy. It follows the methods of the previous analyses (Aartsen et al. 2014c, 2015c) which are improved with respect to the treatment of systematic uncertainties.

The data selection is described in Section 2. The method is described in Section 3. The results of the analysis with respect to the astrophysical signal are presented in Section 4, where we discuss the fit results, tests of alternative hypotheses and investigations on the most energetic event (Schoenen \& Raedel 2015). In Section 5 we present investigations on the directions of recorded events and the attempt to correlate these directions with astrophysical objects. In Section 6 we discuss implications of this analysis for the expected flux of high-energy prompt atmospheric neutrinos from the decay of charmed mesons and obtain the currently most constraining exclusion limit.

\section{DATA SAMPLE}

\subsection{IceCube Detector}

The IceCube Neutrino Observatory is a cubic-kilometersized Cherenkov detector embedded in the ice at the geographic South Pole (Achterberg et al. 2006). It has been designed to detect neutrinos above $\mathrm{TeV}$ energies by measuring the Cherenkov light produced by charged particles produced in neutrino interactions. A total of 5160 optical photomultiplier tubes (PMTs) instrument 86 cable strings with a vertical spacing of $17 \mathrm{~m}$ at depths between 1450 and $2450 \mathrm{~m}$ beneath the surface of the glacial ice sheet (Abbasi et al. 2010). Each PMT is housed in a digital optical module (DOM), consisting of a pressure-resistant sphere, digitization/calibration electronics, and calibration LEDs (Abbasi et al. 2009).

The strings are deployed in a hexagonal pattern with an inter-string spacing of about $125 \mathrm{~m}$ except for the central eight strings which have a smaller spacing of about $60 \mathrm{~m}$ and also a smaller vertical DOM spacing.

The detector was completed in 2010 December; prior to that, data were recorded with partially installed detector configurations. In the remainder of the paper we differentiate the partial detector configurations by the number of strings, e.g., IC59 for the 59-string configuration. The complete detector with 86 strings is referred to by the year the data taking started, e.g., IC2011. The analysis presented here uses data taken from 2009 May until 2015 May which includes the partial detector configurations IC59, IC79 and the seasons IC2011-2014 of the completed detector.

\subsection{Event Selection}

The events that trigger IceCube are predominantly downgoing atmospheric muons produced in cosmic-ray air showers. The standard trigger condition for high-energy neutrino analyses in IceCube requires a minimum of eight DOMs recording light within a time window of $5 \mu \mathrm{s}$, which results in a rate above $2 \mathrm{kHz}$. The triggering DOMs must be in a local 


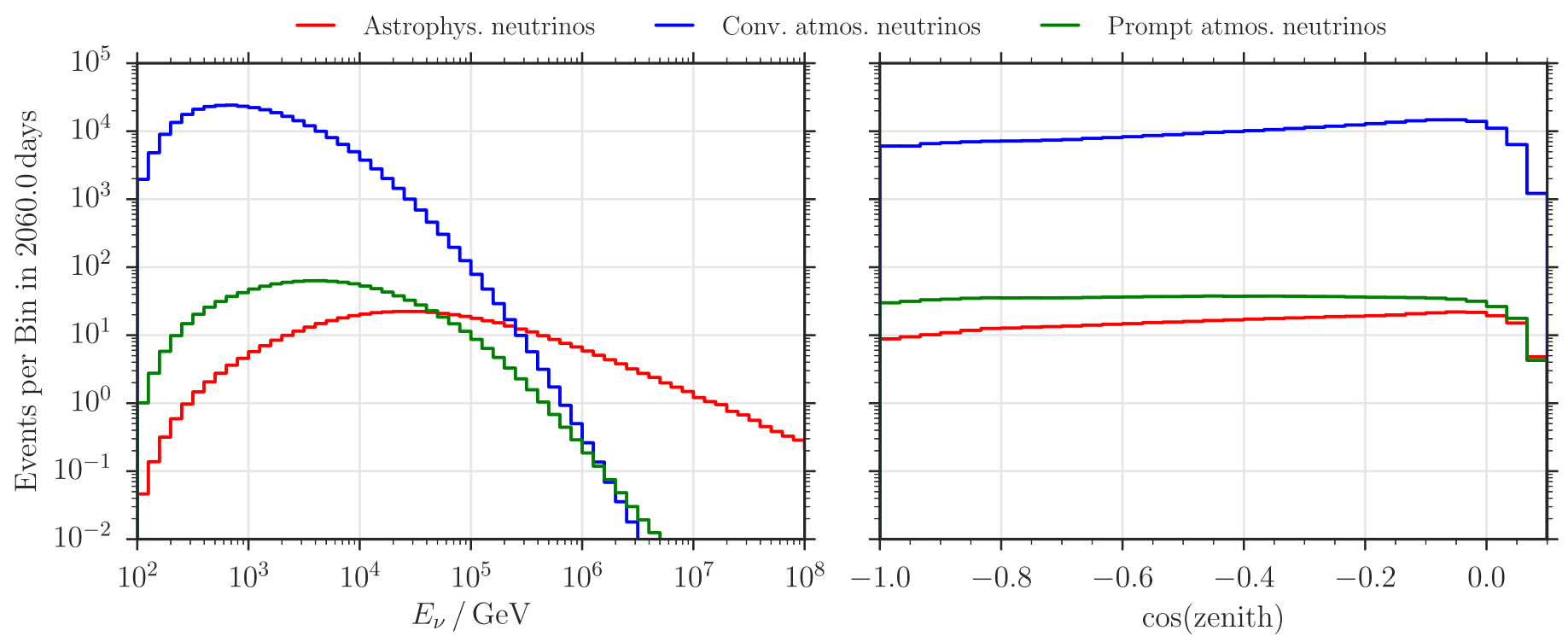

Figure 1. Distribution of the expected neutrino energy (left) and zenith angle (right) for the data selection of this analysis. Shown are the distributions of conventional atmospheric neutrinos (Honda et al. 2007), prompt atmospheric neutrinos (Enberg et al. 2008) where both are corrected for the cosmic-ray spectrum in Gaisser (2012), and a benchmark astrophysical signal $10^{-18} \mathrm{GeV}^{-1} \mathrm{~cm}^{-2} \mathrm{sr}^{-1} \mathrm{~s}^{-1}\left(E_{\nu} / 100 \mathrm{TeV}\right)^{-2}$.

coincidence with either their neighboring or next-to-nearest neighboring DOMs.

For each trigger the digitized PMT waveforms of the detected Cherenkov-light signals are sent to the surface where the number of photons as well as their arrival times are extracted. This information is used to reconstruct the energy and geometry of the event (Ahrens et al. 2004; Abbasi et al. 2013; Aartsen et al. 2014a).

The data processing schemes were improved during the construction of IceCube and the event selection has been optimized for each detector configuration. Data are processed and reconstructed at the South Pole in real time. A filter criterion, optimized for high-energy track-like signatures, requires a minimum amount of detected total charge and a good quality of the track reconstruction. This reduces the data stream to about $34 \mathrm{~Hz}$ that is sent off-site via satellite for further data processing. These events are still dominated by down-going atmospheric muon events. In order to select highenergy up-going muons with high purity and high efficiency, more sophisticated reconstruction algorithms are applied and high-quality events are selected.

The neutrino event selections are based on Monte Carlo (MC) generated neutrinos and atmospheric muons. Note that there are differences in the MC used for the different seasons due to improving simulation code and models which is accounted for in the likelihood fit (see Section 3.2). The simulation of neutrinos is performed by injecting a neutrino at the Earth's surface and propagating it through the Earth. The neutrino interaction in ice or rock is simulated (Gazizov \& Kowalski 2005) with the deep inelastic scattering cross section calculated using the CTEQ5 parton distribution functions (Lai et al. 2000) or the updated HERA1.5 PDFs (Cooper-Sarkar et al. 2011). At the energies of interest the cross sections differ by less than $5 \%$. Each simulated neutrino is forced to interact in the vicinity of the instrumented volume. The volume is scaled as a function of the neutrino energy to include the maximum range of the muon produced in the interaction. The muon is propagated through the detector taking into account energy losses and decay (Chirkin \& Rhode 2004; Koehne et al. 2013).
The Cherenkov light from charged particles is tracked through the ice to the DOMs (Lundberg et al. 2007; Chirkin 2013c, and from work by C. Kopper in $2011^{55}$ ) taking into account the Antarctic ice properties (Ackermann et al. 2006; Aartsen et al. 2013b; Chirkin 2013a). Finally, the detector response and data acquisition are simulated. The same simulation chain is used for atmospheric muons which are simulated with CORSIKA (Heck et al. 1998). Both neutrino simulation and atmospheric muon simulation can be weighted to different fluxes.

The event selection for IC59 is identical to Aartsen et al. (2014c) and covers the up-going zenith range $90^{\circ}-180^{\circ}$. For the later seasons the zenith range has been enlarged to additionally cover angles between $85^{\circ}$ and $90^{\circ}$ as in Aartsen et al. $(2015 \mathrm{c})$ where the overburden by the Antarctic ice sheet is still more than $12 \mathrm{~km}$ of water equivalent. Additionally, the separation of mis-reconstructed atmospheric muons and well reconstructed neutrino-induced muons has been improved by using boosted decision trees (BDTs). For IC2011 and later, the AdaBoost algorithm (Freund \& Schapire 1997) implemented in Pedregosa et al. (2011) has been used. Due to filter and processing changes after the first complete detector season a separate BDT has been trained for IC2011 and the data are treated separately as for the seasons IC59 and IC79.

For the optimization of the BDT we use simulations of upgoing muon neutrinos following an $E^{-2}$ spectrum which produce a muon via a charged-current interaction. In addition, to define the signal for the BDT, only simulated events with directions reconstructed to better than $5^{\circ}$ are used. The background is defined by atmospheric muons from cosmicray air showers that have been mis-reconstructed as up-going. The simulation is weighted to the cosmic-ray model in Gaisser (2012). The features used in the training of the BDT are characteristics of the event topology and parameters evaluating the quality of the reconstructions. These parameters have been selected requiring good agreement between experimental and

\footnotetext{
55 https://github.com/claudiok/clsim
} 

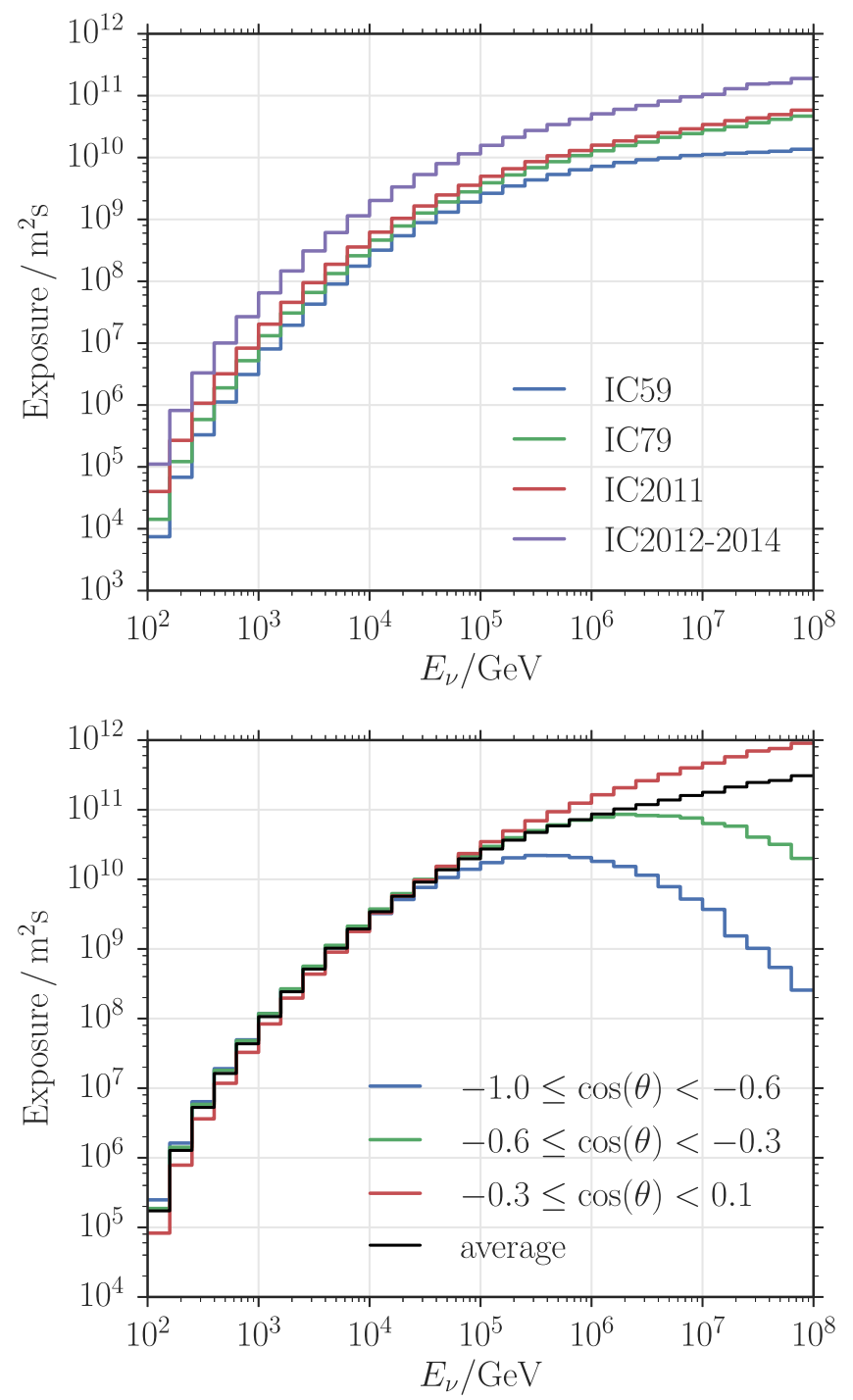

Figure 2. Exposure. Top: individual contributions to the total exposure from the different detector configurations. Bottom: total exposure for different zenith regions for the combined data set. Note that the exposure is based on the sum of the effective areas of $\nu_{\mu}$ and $\bar{\nu}_{\mu}$. Therefore, the total number of events is obtained by integrating the product of exposure and averaged neutrino flux $\Phi_{\left\{\nu_{\mu}+\bar{\nu}_{\mu}\right\} / 2}$ over the neutrino energy and solid angle.

simulated data. The threshold of the BDT classifier is chosen by considering the neutrino selection efficiency and the purity. In order to model the atmospheric background using simulation a high purity is required, rejecting nearly all atmospheric muons. The chosen threshold results in a purity which is better than $99.7 \%$. The remaining background clusters at low energies and is strongly dominated by atmospheric neutrinos. Thus, it cannot affect the analysis and therefore does not have to be taken into account as a separate template in the likelihood fit. The performance estimates are based on 10-fold cross validation (Narsky \& Porter 2013) and a separate validation set. Additionally, a fit of the data has been performed excluding events from above the horizon between $85^{\circ}$ and $90^{\circ}$. Since the fit results remain nearly unaffected we conclude that the fit is not biased by any unaccounted high-energy muons.

Figure 2 shows the total exposure for the different detector configurations and for the full data set for different ranges in cosine zenith. The total number of events as well as the total
Table 1

Summary of the Data Selection

\begin{tabular}{lccc}
\hline \hline Season & $\theta_{\min }-\theta_{\max }(\mathrm{deg})$ & $t_{\text {live }}($ days $)$ & $N_{\text {event }}$ \\
\hline IC59 & $90-180$ & 348.1 & 21,411 \\
IC79 & $85-180$ & 310.0 & 36,880 \\
IC2011 & $85-180$ & 342.1 & 71,191 \\
IC2012-2014 & $85-180$ & 1059.8 & 222,812
\end{tabular}

Note. The table gives the number of events and the effective live time for each data set used.

live time categorized by season are summarized in Table 1. For the best-fit astrophysical flux (see Section 4.2) the expected number of astrophysical muon neutrinos included in these data is approximately 500 .

\section{ANALYSIS METHOD}

\subsection{Likelihood Method}

The experimental and simulated data are binned in two observables sensitive to distinguish between signal and background: reconstructed muon energy and cosine of the zenith angle. These bins are analyzed by a maximum likelihood approach. The expectation in each bin is a function of the signal and nuisance parameters. The likelihood used in this analysis is given by Chirkin (2013b) and is the same as used in Aartsen et al. (2014c). The likelihood per bin is

$$
\mathcal{L}_{i}=\left(\frac{\mu_{i}}{s_{i} / n_{\mathrm{s}}}\right)^{s_{i}} \cdot\left(\frac{\mu_{i}}{d_{i}}\right)^{d_{i}},
$$

where $n_{\mathrm{s}}$ defines the ratio of the live times for simulation and experimental data, $d_{i}$ is the number of events in the data, $s_{i}$ the number of simulated events, and $\mu_{i}$ the expectation in bin $i$. With this likelihood the expectation $\mu_{i}$ is optimized based on the knowledge of the statistics of the simulated and experimental data set. Unlike a Poisson likelihood, we account for the finite statistics of simulated data which becomes relevant for small bin contents in a multi-dimensional parameter space. In the limit of infinite statistics of simulated data this likelihood converges to a saturated Poisson likelihood. A version of Equation (1), modified for weighted events according to Chirkin (2013b), is used in the analysis.

The per-bin expectation is given by

$$
\begin{aligned}
\mu_{i}(\boldsymbol{\theta} ; \boldsymbol{\xi})= & \mu_{i}^{\text {conv. }}(\boldsymbol{\xi}) \\
& +\mu_{i}^{\text {prompt }}\left(\Phi_{\text {prompt }} ; \Delta \gamma_{\mathrm{CR}}, \lambda_{\mathrm{CR}}, \boldsymbol{\xi}_{\mathrm{det}}\right) \\
& +\mu_{i}^{\text {astro. }}\left(\Phi_{\text {astro }}, \gamma_{\text {astro }} ; \boldsymbol{\xi}_{\mathrm{det}}\right),
\end{aligned}
$$

which depends on the signal $\boldsymbol{\theta}$ and nuisance parameters $\boldsymbol{\xi}$ (see Table 2). Here, $\boldsymbol{\xi}_{\text {det }}$ corresponds to the parameters taking into account the neutrino detection uncertainties (see Section 3.2.1). In this analysis, the signal parameters consist of the astrophysical flux parameters and the prompt flux parameter. The astrophysical flux model used here is a single power-law flux described by two parameters: the normalization $\Phi_{\text {astro }}$ at $100 \mathrm{TeV}$ neutrino energy and the spectral index $\gamma_{\text {astro }}$ :

$$
\Phi_{\nu+\bar{\nu}}=\Phi_{\text {astro }} \cdot\left(\frac{E}{100 \mathrm{TeV}}\right)^{-\gamma_{\text {astro }}}
$$


Table 2

Nuisance Parameters

\begin{tabular}{|c|c|c|c|c|c|c|}
\hline & IC59 & IC79 & IC2011 & IC2012-2014 & scaling & Best Fit (68\% C.L.) \\
\hline \multicolumn{7}{|l|}{ Flux properties: } \\
\hline Conventional flux $\Phi_{\text {conv }}$ & 1.028 & 1.047 & 1.184 & 1.194 & relative & $0.998 \pm 0.003$ \\
\hline Kaon-pion ratio $\mathrm{K} / \pi$ & 1.310 & 1.514 & 1.002 & 1.032 & relative & $0.977 \pm 0.027$ \\
\hline Cosmic-ray model $\lambda_{\mathrm{CR}}$ & $1.0(\mathrm{H} 3 \mathrm{p})$ & $1.0(\mathrm{H} 3 \mathrm{p})$ & $1.0(\mathrm{H} 3 \mathrm{p})$ & $1.0(\mathrm{H} 3 \mathrm{p})$ & absolute & $0.0-0.5$ \\
\hline Optical efficiency $\epsilon_{\mathrm{opt}}$ & 1.011 & 0.974 & 1.042 & 1.056 & relative & $1.002 \pm 0.002$ \\
\hline Absorption length $\lambda_{\text {abs }}$ & $\cdots$ & $\cdots$ & 1.000 & 1.047 & relative & $1.001 \pm 0.004$ \\
\hline Absorption/scattering length $\lambda_{\text {abs/scat }}$ & $\cdots$ & 0.991 & $\cdots$ & $\cdots$ & relative & $1.000 \pm 0.004$ \\
\hline Ice model $\lambda_{\text {icel }}[$ SpiceMie,WHAM] & 0. (SpiceMie) & SpiceMie (fixed) & $\cdots$ & $\cdots$ & absolute & $0.000+0.014$ \\
\hline Ice model $\lambda_{\text {ice2 }}$ [SpiceMie,SpiceLea] & $\cdots$ & $\cdots$ & 0.551 & SpiceLea (fixed) & absolute & $0.006 \pm 0.057$ \\
\hline
\end{tabular}

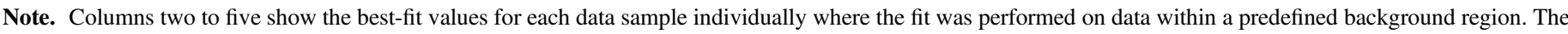

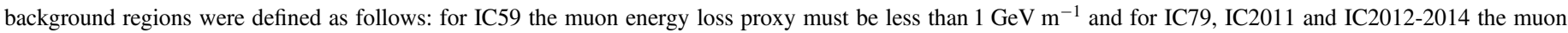

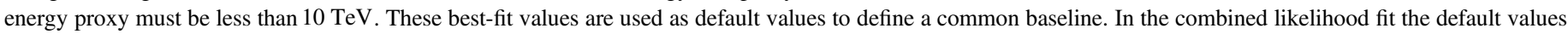

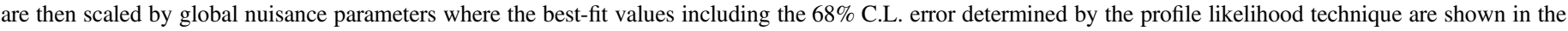

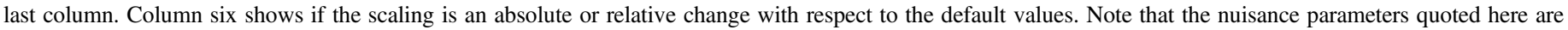

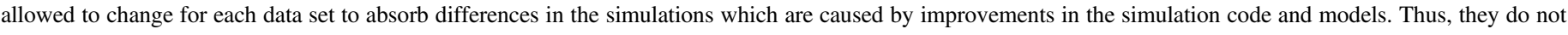
have to reflect their real physical quantities.

The prompt neutrino flux is described by the prediction taken from Enberg et al. (2008) (ERS) where the absolute normalization $\Phi_{\text {prompt }}$ is taken as free parameter. In addition, nuisance parameters are introduced to take into account systematic uncertainties, e.g., the conventional atmospheric neutrino flux is described by the prediction taken from Honda et al. (2007) and the flux normalization $\Phi_{\text {conv }}$ is taken as a nuisance parameter. Note that the conventional and prompt neutrino flux predictions have been corrected for the knee in the cosmicray spectrum based on the cosmic-ray models in Hoerandel (2003) and Gaisser (2012) (see Section 3.2). The implementation of nuisance parameters is described in Section 3.2 in more detail.

The global likelihood, which is maximized, is the product of all per-bin-likelihoods $\mathcal{L}=\prod_{i} \mathcal{L}_{i}$. The significances and parameter uncertainties in this analysis are derived using the profile likelihood technique and Wilks' theorem (Wilks 1938). The applicability of Wilks' theorem has been tested and verified by ensemble studies.

\subsection{Systematic Uncertainties}

In order to account for systematic uncertainties, resulting from the imperfect background and signal modeling, continuous nuisance parameters valid for the entire energy and zenith range are introduced. The systematic uncertainties can be divided into two categories: neutrino detection uncertainties and atmospheric flux uncertainties. The former include the optical efficiency of the detector, the neutrino-nucleon cross section, the muon energy loss cross section and the optical properties of the Antarctic ice. The latter include the flux normalizations, the spectral shape and composition of the cosmic-ray spectrum in the "knee" region, the spectral index of the primary cosmic-ray spectrum, and the relative production yield of pions and kaons in the atmosphere. The implementation of these uncertainties as nuisance parameters in the likelihood function is done similar to Aartsen et al. (2014c). Main improvements with respect to previous analyses are the parameterizations of the systematic detector effects as unbinned functions of both fit observables (S. Schoenen 2016, in preparation) using adaptive kernel density estimation and the interpolations between specific models to account for the model uncertainties. The systematic detector effects are studied by simulated data sets where the default parameters are changed within their uncertainties. In cases where no interpolation between specific models is used, the nuisance parameters are implemented by using independent correction factors $f_{k}\left(\xi_{k}\right)$. These factors scale the default per-bin expectation $\mu_{i}^{0}$ for each flux contribution with respect to the individual nuisance parameter $\xi_{k}$ :

$$
\mu_{i}^{0} \mapsto \mu_{i}^{0} \cdot \prod_{k} f_{k}\left(\xi_{k}\right)
$$

\subsubsection{Neutrino Detection Uncertainties}

Optical efficiency of the detector. The optical efficiency $\epsilon_{\mathrm{opt}}$ takes into account all uncertainties related to the light production and detection in the detector, e.g., the number of produced Cherenkov photons, the overall optical transparency of the ice, the photon detection efficiency of the DOMs, and the shadowing of photons by detector components. Since the optical efficiency is directly connected to the brightness of an event as observed with the detector, its uncertainty results in an uncertainty on the reconstructed energy scale. The effect has been parameterized as a function of the muon energy proxy and the cosine zenith angle and is implemented as a nuisance parameter. The uncertainty on the optical efficiency is estimated to be less than $15 \%$. Since the ice properties of the refrozen water within the drill holes differ from the bulk ice properties they are taken into account as a modification of the angular acceptance (Aartsen et al. 2013b).

Optical properties of the Antarctic ice. The probability of a Cherenkov photon to be detected by a DOM depends not only on the optical efficiency of the detector but also on the optical transparency of the Antarctic ice. The main processes are 
scattering and absorption of photons on their path to the DOM. For the Antarctic ice this is modeled by depth-dependent scattering and absorption lengths. The modeling is done using measured data from calibration light sources that are integrated into the DOMs. Different models of the ice have been developed during the operation of IceCube. For this analysis the following ice models are used: WHAM for IC59 (based on a measurement of the optical properties of the glacial ice at the South Pole presented in Ackermann et al. 2006), SpiceMie for IC59, IC79 and IC2011 (Aartsen et al. 2013b), and SpiceLea for IC2011-2014 (Chirkin 2013a). For all available simulation data sets, the effects of the optical ice properties as a function of reconstructed energy proxy and cosine zenith are parameterized and implemented as a nuisance parameter. This is done for different ice models and each detector configuration. The parameterization is done by introducing a parameter $\lambda_{\text {ice }}$ that describes a linear combination $\left(\lambda_{\text {ice }} \cdot \mathrm{M}_{1}+\left(1-\lambda_{\text {ice }}\right) \cdot \mathrm{M}_{2}\right)$ between two ice models where $\mathrm{M}_{1}$ and $\mathrm{M}_{2}$ are the expectations per bin corresponding to the two ice models. In addition, for a given ice model the effects of different scattering lengths $\lambda_{\text {scat }}$ and absorption lengths $\lambda_{\text {abs }}$ on the muon energy proxy and the cosine zenith angle have been parameterized. Owing to missing simulations this could not be done for IC59. For IC79 the scattering and absorption lengths have been varied simultaneously resulting in only one effective nuisance parameter $\lambda_{\text {abs/scat }}$. From IC2011 on the scattering and absorption lengths have been varied separately. For more information see Table 2. The individual uncertainty for both quantities is estimated to be less than $10 \%$. The scattering length mainly influences the angular resolution of the neutrino arrival direction and therefore the reconstructed zenith angle. Since the cosine zenith bin width of the analysis is relatively coarse, the effect of this uncertainty on the observable distribution is small. The absorption length mainly influences the flux normalization and the shape of the energy distribution. This effect is much larger, compared to the scattering length effect.

\subsubsection{Atmospheric Flux Uncertainties}

Flux normalization. The uncertainty on the normalization of the conventional atmospheric neutrino flux is implemented as a nuisance parameter $\Phi_{\text {conv }}$ that scales the flux normalization of the model by Honda et al. (2007). This model has been extrapolated to higher energy based on the method in taking into account a more realistic spectrum of cosmic rays and their composition (Illana et al. 2011; Schukraft 2013). Note that the uncertainty of this parameter is relatively large, on the order of $30 \%$. Thus, it absorbs any kind of uncertainty which influences the global flux normalization in the fit.

Cosmic-ray model and spectral index. The composition of the cosmic rays is uncertain, in particular above the knee at an energy of about $3 \mathrm{PeV}$. Models are based on the superposition of Galactic cosmic-rays with rigidity-dependent cut-offs and an emerging extragalactic component. Since conventional and prompt atmospheric neutrinos are produced by cosmic-ray interactions within the atmosphere, the uncertainty on the cosmic-ray spectrum also affects the expectation of these neutrinos. The effect of different cosmic-ray models is parameterized by $\lambda_{\mathrm{CR}}$ as a function of the muon energy proxy and the cosine zenith angle similar to the discrete ice models. Here, a linear combination between Hoerandel (2003) and Gaisser (2012), which are the extreme cases, is used. In addition to the effects between different cosmic-ray models an overall change in the cosmic-ray spectral index affects the expectation of atmospheric neutrinos. Therefore, a shift of the cosmic-ray spectral index $\Delta \gamma_{\mathrm{CR}}$ is implemented as a nuisance parameter representing the uncertainty on the cosmic-ray spectral index. The uncertainty is estimated to be of the order of $4 \%$ based on differences between the aforementioned cosmic-ray models. A positive $\Delta \gamma_{\mathrm{CR}}$ corresponds to a softer energy spectrum.

Kaon-to-pion ratio. Conventional atmospheric neutrinos are produced by decays of pions and kaons which are themselves produced in air showers. The relative contribution of kaons and pions $\mathrm{K} / \pi$ to the production of conventional atmospheric neutrinos affects their zenith angle distribution. In this analysis it is defined by the ratio of the integrated neutrino fluxes from kaon and pion decays. Using the neutrino flux parameterization from Gaisser (1990) fitted to the conventional atmospheric neutrino prediction from Honda et al. (2007) between 1 and $10 \mathrm{TeV}$, the kaon-to-pion ratio is implemented as a nuisance parameter where the uncertainty is estimated to be of the order of $10 \%$.

Atmospheric temperature effects. The expected number of conventional atmospheric neutrinos is directly connected to the number of pion and kaon decays in the atmosphere. A denser atmosphere will increase the interaction probability for pions and kaons relative to the decay probability, which reduces the overall neutrino flux. Thus, annual temperature fluctuations influence the expectation of conventional atmospheric neutrinos. Since the prediction of conventional atmospheric neutrinos from Honda et al. (2007) is based on the standard US atmosphere, the expectation is corrected for annual temperature fluctuations. This is done using the formalism reported in Desiati et al. (2014) and data measured by the instrument AIRS installed on the AQUA satellite (AIRS Science Team/Joao Texeira 2013). The effect of this correction is estimated to be of the order of $2 \%$ with an uncertainty of about $0.1 \%$.

With the goal of achieving a unbiased result for the signal parameters, we note that many nuisance parameters were deliberately chosen correlated (see Figure 3 ). For example, the optical detector efficiency is correlated to the Cherenkov light yield uncertainty and to the effects of the uncertainties in the muon energy loss cross sections. In cases where the effects of one uncertainty are fully absorbed by other nuisance parameters only one parameter has been implemented for better numerical stability of the fit. A list of the implemented parameters is given in Table 2. In order to obtain an unbiased result the nuisance parameters are implemented without priors which is tested to have no effect on the sensitivity for an astrophysical or prompt flux.

The fit procedure was tested by generating pseudo experiments where the input parameters were varied. The fit of the signal parameters was found to be robust and unbiased against the choice of nuisance parameters.

Since IceCube's MC simulations have evolved and improved from year to year, the default expectations for each nuisance parameter have changed for simulated data sets year by year. In order to avoid a tension in the fitted nuisance parameters induced purely by the differences of the simulations, two methods were tested: the implementation of individual nuisance parameters for each year, and the alignment of all nuisance parameters to a common baseline. We found that the two methods give similar sensitivities both for the astrophysical and the prompt flux parameters, and the 


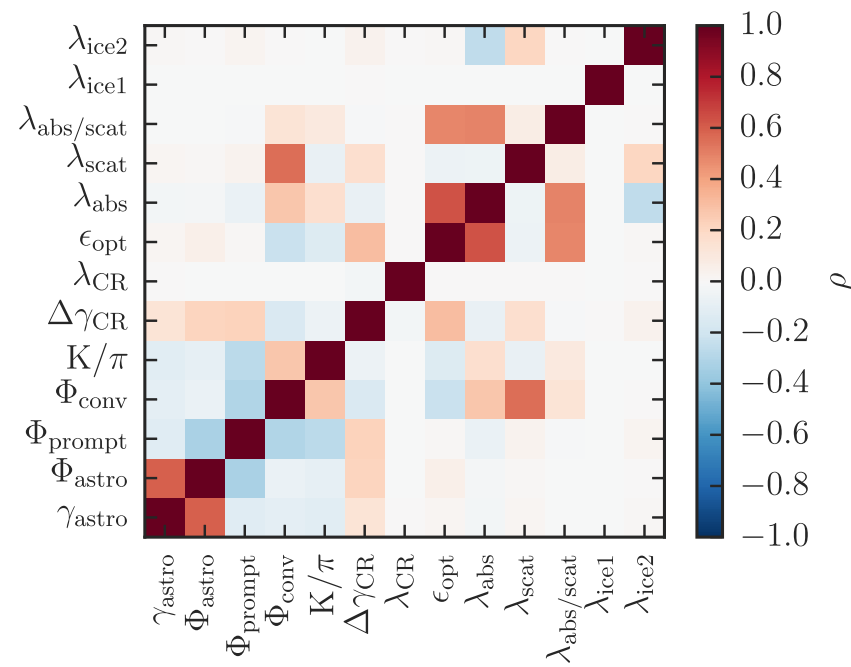

Figure 3. Correlation matrix of signal and nuisance parameters. $\lambda_{\text {ice } 1}$ describes the linear combination between SpiceMie (Aartsen et al. 2013b) and WHAM (Ackermann et al. 2006) for IC59, $\lambda_{\text {ice2 }}$ describes the linear combination between SpiceMie (Aartsen et al. 2013b) and SpiceLea (Chirkin 2013a) for IC2011. $\lambda_{\text {abs/scat }}$ describes the relative change of the ice properties for IC79 and $\lambda_{\text {abs }}, \lambda_{\text {scat }}$ describes the relative change of the ice properties for IC2011-14.

alignment method is chosen since the time consumption for the fit is much lower. The alignment is done by fitting the nuisance parameters in a predefined background region for each year individually. The resulting best-fit values for the nuisance parameters, summarized in Table 2, define the default values for each year. In the combined likelihood fit of all six years these default values are then scaled by global nuisance parameters. The scaling can be either an absolute or relative change with respect to the aligned default values.

The flavor composition at Earth is not identical to the flavor composition at an astrophysical source due to neutrino oscillations. For a source dominated by $p \gamma / p p$-interactions, the initial flavor ratio of 1:2:0 $\left(\nu_{e}: \nu_{\mu}: \nu_{\tau}\right)$ is transformed to be approximately equally partitioned among the flavors (Learned \& Pakvasa 1995; Athar et al. 2006). Therefore, it is necessary to take into account the muonic decay of taus originating from charged current $\nu_{\tau}$ interactions by combining it with the astrophysical muon neutrino flux. A fit of the data with and without this contribution shows that accounting for it leads to a decrease in the astrophysical normalization of about $5 \%$ and has no effect on the spectral index. In the rest of the paper we account for the contribution of $\nu_{\tau}$ assuming equal partitioning. The astrophysical normalization for other expected flavor compositions of astrophysical neutrino sources can be obtained by rescaling.

\subsection{Parametric Unfolding}

The best-fit result for the neutrino energy spectrum measured by this analysis can be used to determine for each event a neutrino energy probability density function $P\left(E_{\nu} \mid E_{\text {reco }}^{i}\right)$ with respect to its muon energy proxy $E_{\text {reco. These functions depend }}^{i}$ on the assumption for the neutrino energy spectrum and are therefore model-dependent. In particular, for the astrophysical neutrino flux an unbroken, single power law is assumed. For the full six-year data sample the neutrino energy distribution is given by the sum over the probability density function of all events $\sum_{i} P\left(E_{\nu} \mid E_{\text {reco }}^{i}\right)$ where each function is normalized to an event count of one. Since this approach is model-dependent it is called parametric unfolding in the following. Note that this method cannot replace a model-independent unfolding as done in Boerner et al. (2015) for different IceCube data samples.

\section{RESULTS OF THE SPECTRAL LIKELIHOOD FIT}

\subsection{Fit Result}

The result of the fit is presented as a set of one-dimensional projections of energy and zenith in Figure 4 separately for each contributing data sample. The experimental data are shown as black crosses, and the best-fit expectations for astrophysical and conventional atmospheric neutrinos are shown as red and blue bands, respectively. An excess of high-energy events consistent with an astrophysical signal above the atmospheric background is visible for each data sample. The overall agreement between the data and the MC of the full data set is good for all energies and zenith angles. We have tested quality of the fit based on the two-dimensional distributions using the ratio between the likelihood (Equation (1)) and the saturated likelihood as test-statistic (Olive et al. 2014). The test-statistic distribution was generated via pseudo experiments based on the best fit. The resulting $p$-value is $95.4 \%$, indicating a very good agreement between data and MC. All nuisance parameters are fitted to values consistent with their uncertainty (Table 2).

Note that the data sample taken with the 79-string configuration contains roughly twice as many high-energy events above a reconstructed muon energy of $100 \mathrm{TeV}$ than other years (see Figure 4). Nevertheless, the result of the fit for all years is consistent with the fits for individual years. Visual inspection and other cross checks of these events revealed no indication of any time-dependent detector effects. A dedicated analysis searching for time-dependent neutrino sources (Aartsen et al. 2015e) has found no indication of a signal. Therefore, the observations are consistent with a statistical fluctuation.

\subsection{Astrophysical Flux}

The best fit for the unbroken power-law model of the astrophysical flux results in

$$
\Phi_{\nu+\bar{\nu}}=\left(0.90_{-0.27}^{+0.30}\right) \cdot\left(E_{\nu} / 100 \mathrm{TeV}\right)^{-(2.13 \pm 0.13)}
$$

in units of $10^{-18} \mathrm{GeV}^{-1} \mathrm{~cm}^{-2} \mathrm{sr}^{-1} \mathrm{~s}^{-1}$. The statistical significance of this flux with respect to the atmospheric-only hypothesis is 5.6 standard deviations. The fit results are shown in Figure 5 and summarized in Table 3. The quoted errors are based on the profile likelihood using Wilks' theorem (Wilks 1938) and include both statistical and systematic uncertainties. No contribution from prompt atmospheric neutrinos is preferred by the best-fit spectrum, and an upper limit, based on the profile likelihood, is shown in Figure 5. For more information about the upper limit for prompt atmospheric neutrinos see Section 6.

The two-dimensional contours of the profile likelihood as a function of the signal parameters are shown in Figure 6. While the fitted astrophysical flux normalization is strongly correlated with the astrophysical spectral index, these astrophysical signal parameters are found to be largely independent of the prompt flux normalization.

The model assumes an unbroken power law for the astrophysical signal. We estimate that neutrinos in the 

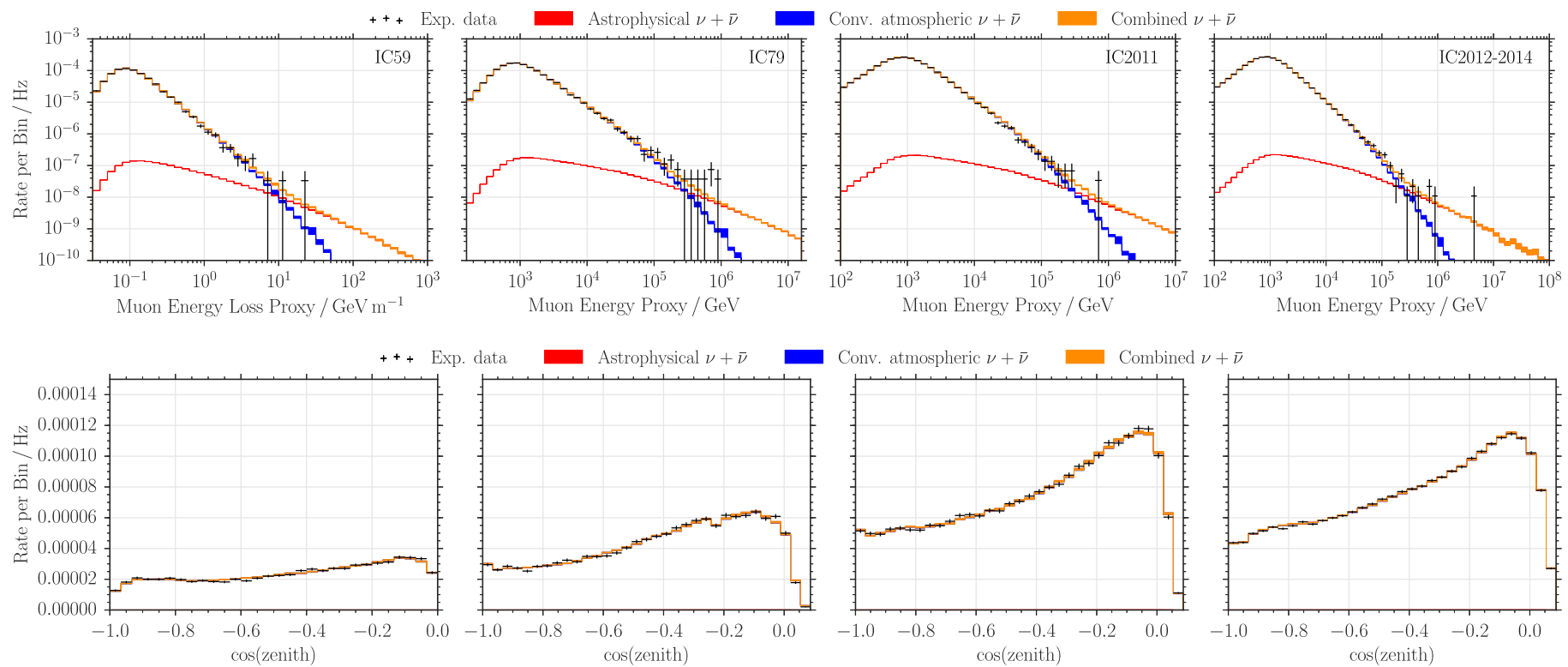

Figure 4. Distributions of the experimental data for the muon energy proxy (top) and reconstructed zenith (bottom) for each contributing data sample (left: 59-string, center-left: 79-string, center-right: 86-string (2011-2012), right: 86-string (2012-2015)). Note that IC2011 is different from the later years due to changes in the data processing. The best-fit model for astrophysical and atmospheric neutrinos is superimposed. Only statistical errors are shown.

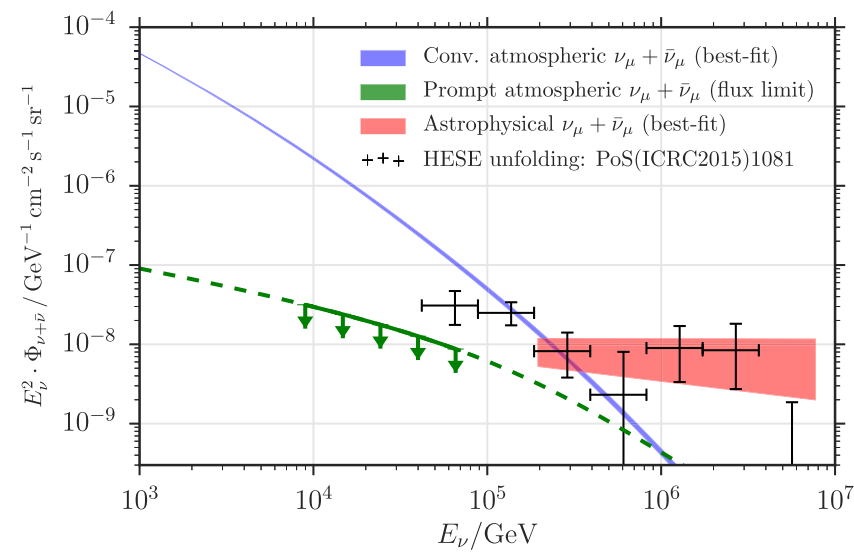

Figure 5. Best-fit neutrino spectra for the unbroken power-law model. The width of the line corresponding to conventional atmospheric neutrinos (blue) represents the one-sigma error on the measured spectrum. The width of the line corresponding to astrophysical neutrinos (red) shows the effect of varying both of the astrophysical parameters within one-sigma of the best fit values, without accounting for correlation. The green line represents the upper limit on the prompt model (Enberg et al. 2008). The horizontal width of the red band denotes the energy range of neutrino energies which contribute $90 \%$ to the total likelihood ratio between the best-fit and the conventional atmospheric-only hypothesis. The black crosses show the unfolded spectrum published in Kopper et al. (2015).

experimental data sample with energies mainly between $194 \mathrm{TeV}$ and $7.8 \mathrm{PeV}$ contribute to this observation. This energy range is shown in Figure 5. It defines the central range of neutrino energies that contribute $90 \%$ to the total observed likelihood ratio between the best fit and the conventional atmospheric-only hypothesis. Note that this definition is different from Aartsen et al. (2015b, 2015c).

\subsection{Multi-PeV Track-like Event}

The selected data include one exceptionally high-energy muon event that is shown in Figure 7 (Schoenen \& Raedel 2015). The deposited energy has been measured to $(2.6 \pm 0.3)$
Table 3

Best-fit Parameter Values for the Unbroken Power-law Model

\begin{tabular}{lcc}
\hline \hline Parameter & Best-Fit & $68 \%$ C.L. \\
\hline$\Phi_{\text {astro }}$ & 0.90 & $0.62-1.20$ \\
$\gamma_{\text {astro }}$ & 2.13 & $2.00-2.26$ \\
$\Phi_{\text {prompt }}$ & 0.00 & $0.00-0.19$ \\
\hline
\end{tabular}

Note. $\Phi_{\text {astro }}$ is the normalization of the astrophysical neutrino flux at $100 \mathrm{TeV}$ and is given in units of $10^{-18} \mathrm{GeV}^{-1} \mathrm{~s}^{-1} \mathrm{sr}^{-1} \mathrm{~cm}^{-2}$. $\Phi_{\text {prompt }}$ is given in units of the model in Enberg et al. (2008). The normalizations correspond to the sum of neutrinos and antineutrinos.

$\mathrm{PeV}$ of equivalent electromagnetic energy Aartsen et al. (2014a). Assuming the best-fit atmospheric energy spectrum from this analysis (see Figure 5) the $p$-value of this event being of atmospheric origin has been estimated to be less than $0.005 \%$, strongly suggesting an astrophysical origin.

The segmented energy loss reconstruction described in Aartsen et al. (2014a) can be used to reconstruct the direction of through-going muons. This includes the timing of not only the first photon but all photons as well as the total number of photons. The reconstructed direction of the event is given in Table 4 and discussed in Section 5.1.

In order to estimate the angular uncertainty and the most likely muon and neutrino energy we have simulated events with energies according to our best-fit energy spectrum with directions varying by $1^{\circ}$ around the best-fit direction. Additionally, the position where the muon enters the instrumented volume has been varied within $10 \mathrm{~m}$. Systematic uncertainties due to the lack of knowledge of the optical ice properties are taken into account by varying the ice model parameters within their uncertainties during the simulation.

Based on these simulations we evaluate the muon energy at the point of entrance into the instrumented volume, that results in the observed deposited energy. The obtained median muon energy is $(4.5 \pm 1.2) \mathrm{PeV}$ where the error range corresponds to $68 \%$ C.L. 

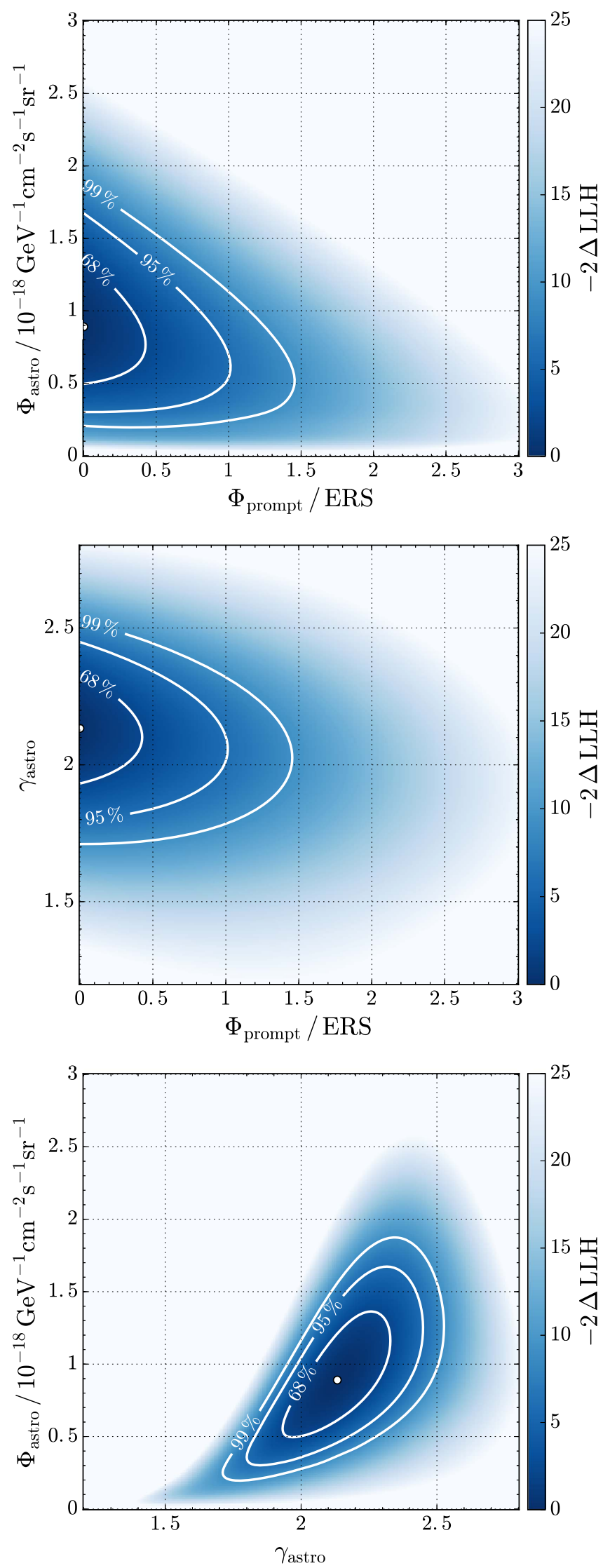

Figure 6. Two-dimensional profile likelihood scans of the astrophysical parameter $\Phi_{\text {astro }}, \gamma_{\text {astro }}$ and the prompt normalization $\Phi_{\text {prompt }}$ in units of the model in Enberg et al. (2008). The contours at 68\%, 95\%, and 99\% CL assuming Wilks' theorem are shown.
For the estimation of the median expected neutrino energy we have taken into account that high-energy muons arise not only from $\nu_{\mu}$ charged-current interactions but also from muonic decay of charged-current $\nu_{\tau}$ interactions and muonic $W^{-}$decays in $\bar{\nu}_{e}+e^{-} \rightarrow W^{-}$interactions. Here, we assume the best-fit astrophysical spectrum and an equal flux of all flavors but include the effects of the Earth's absorption for the specific decl. of the event. Under these assumptions, we find $87.7 \%$ probability of a primary $\nu_{\mu}, 10.9 \%$ for a primary $\nu_{\tau}$ and $1.4 \%$ for a primary $\bar{\nu}_{e}$. The respective probability distributions of primary neutrino energy are shown in Figure 8. The expected neutrino energy depends on the primary flavor. The median expected muon neutrino energy is $8.7 \mathrm{PeV}$ for the above assumptions.

The angular reconstruction uncertainty including systematic uncertainties of the Antarctic ice (see Section 3.2) can be estimated from the aforementioned dedicated simulation. Figure 9 shows the angular reconstruction uncertainty for an ensemble of events with similar deposited energy, direction and entry point into the fiducial volume. The angular reconstruction uncertainty is given by the angular distance between the true and the reconstructed muon direction. The median angular uncertainty is $0.23^{\circ}$ and the $99 \%$ containment is $0.9^{\circ}$. Details of the studies of the multi-PeV track-like event are shown in L. Rädel (2016, in preparation).

\subsection{Test for a Spectral Cut-off}

The default hypothesis of an unbroken power law is tested against the hypothesis of a spectral cut-off. For this, an exponential energy cut-off $E_{\nu}^{\text {cut-off }}$ is added to the astrophysical neutrino flux:

$$
\Phi_{\nu+\bar{\nu}}=\Phi_{\text {astro }} \cdot \exp \left(-\frac{E_{\nu}}{E_{\nu}^{\text {cut }- \text { off }}}\right) \cdot\left(\frac{E_{\nu}}{100 \mathrm{TeV}}\right)^{-\gamma_{\text {astro }}} .
$$

In the fit the spectral index $\gamma_{\text {astro }}$ is highly degenerate with an exponential energy cut-off $E_{\nu}^{\text {cut-off }}$, therefore two scenarios with fixed spectral indices have been tested. For the spectral indices the benchmark model with $\gamma_{\text {astro }}=2$ and the best-fit value $\gamma_{\text {astro }}=2.13$ are chosen. Figure 10 shows the twodimensional contours of the profile likelihood as a function of the signal parameters $\Phi_{\text {astro }}, E_{\nu}^{\text {cut-off }}$ and $\Phi_{\text {prompt }}$. For the benchmark model a cut-off is slightly preferred at the level of one standard deviation. This is an expected behavior as the actual best-fit spectral index is softer. Thus, fixing the spectral index to a harder spectrum will result in a slight deficit at the highest neutrino energies. When fixing the spectral index to the best-fit value for an unbroken power law, this slight preference for an exponential cut-off disappears. These results are nearly independent of the prompt flux normalization.

\subsection{Unfolded Astrophysical Spectrum}

The best-fit results for the neutrino energy spectrum as quoted in Table 3 and the knowledge of the connection between the reconstructed muon and true neutrino energy can be used to unfold a neutrino energy distribution for the six-year sample (see Section 3.3). The results of this parametric unfolding are shown in Figure 11 as a cumulative energy distribution of the number of neutrinos with energies greater than $E_{\nu}$. The statistical error band is given by the square root of this number. The error band that corresponds to the uncertainty 


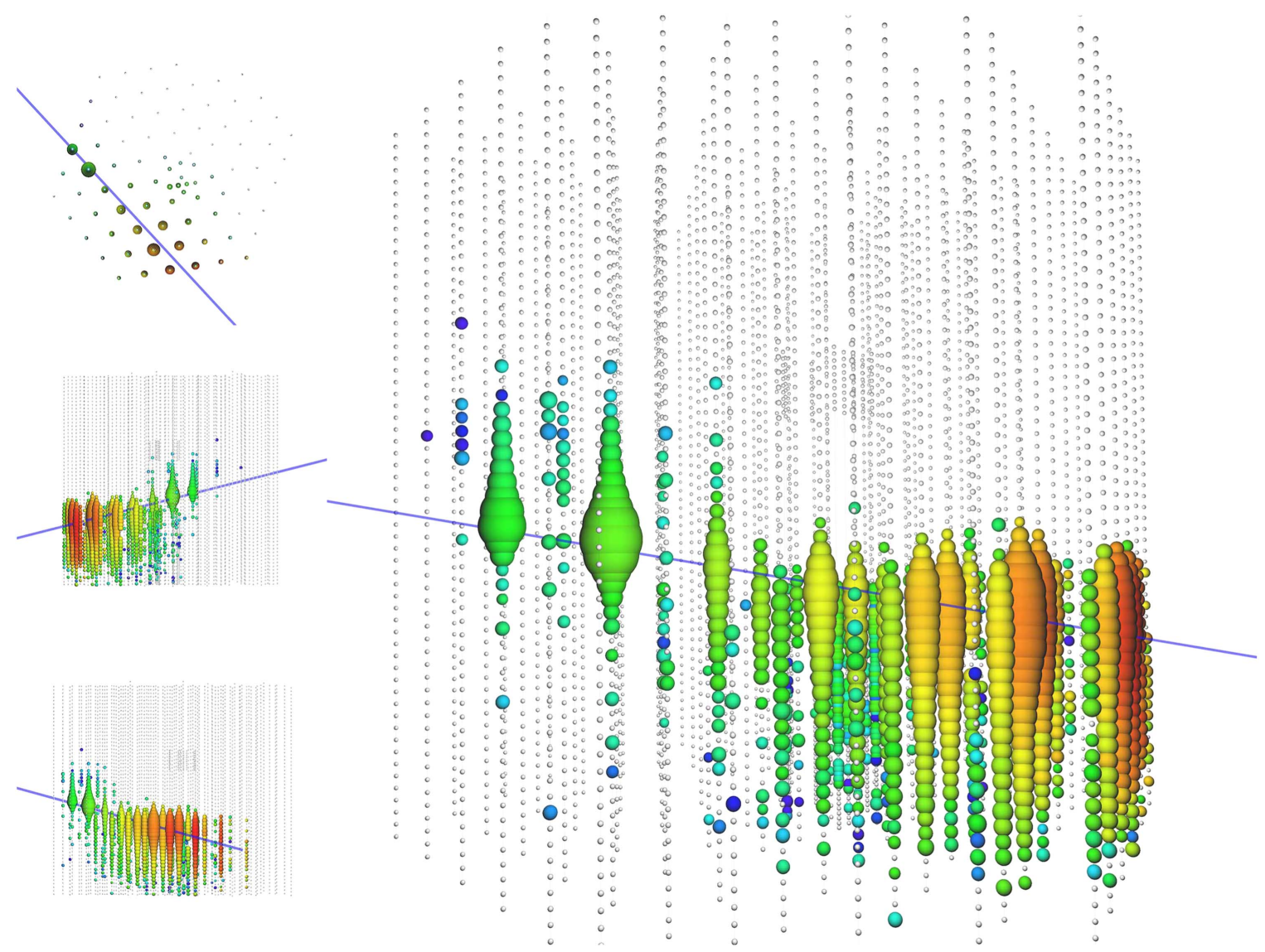

Figure 7. Event view of the PeV track-like event recorded by IceCube on 2014 June 11. Left: top and two side views. Right: perspective view. Shown are the IceCube DOMs as black dots. The colors indicate the photon arrival time from red (early) to green (late) and the size of the sphere the amount of measured charge. Note that the scaling is nonlinear and a doubling in sphere size corresponds to one hundred times the measured charge. The blue line shows the reconstructed particle track. The reconstructed equatorial coordinates of this event are decl. $=11^{\circ} .42$ and R.A. $=110^{\circ} .63$. This event deposited an energy of $2.6 \pm 0.3 \mathrm{PeV}$ within the detection volume.

on the astrophysical flux is determined by varying the astrophysical spectrum within the measured uncertainties on the astrophysical flux parameters. Based on the per-event probability density function $P\left(E_{\nu} \mid E_{\text {reco }}^{i}\right)$ the median neutrino energy for each event can also be calculated. Figure 12 shows the distribution of the median neutrino energies for the six-year sample.

In both distributions a clear excess above approximately $100 \mathrm{TeV}$ in neutrino energy is visible, and is not compatible with the atmospheric background expectation. Although only a single event with energy greater than a PeV has been observed, we can infer from our fit and from the relation between muon energy and energy of the parent neutrino that there are most likely several neutrinos with energies above a PeV in the sixyear sample.

\subsection{Discussion}

This analysis found an astrophysical spectral index of $\gamma=2.13 \pm 0.13$, which is harder than previously reported measurements, see e.g., Kopper et al. (2015), Neiderhausen et al. (2015) and Aartsen et al. (2015f). We refer to these analyses in the rest of the section as starting event analysis, cascade analysis and combined analysis, respectively. Figure 13 compares the measured astrophysical neutrino flux normalization and spectral index with these results and the previous measurement using through-going muons Aartsen et al. (2015c).
While the sample used in the cascade analysis is completely statistically independent, the starting event analysis and global fit have an overlap in events. The combined fit includes three years of muon data from 2009 to 2012 based on Aartsen et al. (2014c, 2015c). The starting event analysis includes a small fraction $(6 \%)$ of up-going muons that start within the detector, that are also included here. However, these three analyses are strongly dominated by independent cascade-like events of which a large fraction originates from the Southern Hemisphere. For the starting event analysis $73 \%$ of the events above $100 \mathrm{TeV}$ are down-going and $93 \%$ of these are cascade-like. For the investigation of the tension in the observed energy spectrum of astrophysical neutrinos, the assumption of statistical independence is reasonably well justified but will result in a lower limit on the tension.

The combined analysis finds the smallest confidence region of the three aforementioned results. The $p$-value for obtaining the combined fit result and the result reported here from an unbroken powerlaw flux is $3.3 \sigma$, and is therefore in significant tension. For the discussion, it is important to highlight the systematic differences between these measurements. The threshold for the up-going muon signal is a few hundred $\mathrm{TeV}$ while astrophysical starting events are detected above a few times $10 \mathrm{TeV}$. It should be noted that for the overlapping energy region $>200 \mathrm{TeV}$ the measured fluxes for the cascadedominated channels are in good agreement with the results reported here, as shown in Figure 5. As a conclusion, we 
Table 4

Summary of Highest Energy Events above $200 \mathrm{TeV}$ in All Years

\begin{tabular}{|c|c|c|c|c|c|c|c|c|c|}
\hline ID & MJD & Signalness & Energy Proxy $(\mathrm{TeV})$ & Decl. (deg) & $50 \%$ C.L. & 90\% C.L. & R.A. (deg) & $50 \%$ C.L. & $90 \%$ C.L \\
\hline 1 & $55056.70^{\mathrm{a}}$ & 0.78 & 480 & 1.23 & $\begin{array}{l}0.08 \\
-0.08\end{array}$ & $\begin{array}{l}+0.18 \\
-0.22\end{array}$ & 29.51 & $\begin{array}{l}0.15 \\
{ }_{-0.17}\end{array}$ & $\begin{array}{l}{ }_{-0.38}^{+0.40} \\
\end{array}$ \\
\hline 2 & $55141.13^{\mathrm{a}}$ & 0.52 & 250 & 11.74 & ${ }_{-0.18}^{+0.10}$ & $\begin{array}{l}+0.32 \\
-0.38\end{array}$ & 298.21 & ${ }_{-0.22}^{+0.17}$ & $\begin{array}{l}+0.53 \\
-0.57\end{array}$ \\
\hline 3 & $55355.49^{\mathrm{b}}$ & 0.65 & 340 & 23.58 & $\begin{array}{l}+0.91 \\
{ }_{-1.18}\end{array}$ & ${ }_{-4.13}^{+2.31}$ & 344.93 & ${ }_{-1.04}^{+1.14}$ & $\begin{array}{l}+3.39 \\
{ }_{-2.90}\end{array}$ \\
\hline 4 & $55370.74^{\mathrm{b}}$ & 0.54 & 260 & 47.80 & ${ }_{-0.22}^{+0.25}$ & $\begin{array}{l}+0.56 \\
-0.48\end{array}$ & 141.25 & ${ }_{-0.16}^{+0.23}$ & ${ }_{-0.45}^{+0.46}$ \\
\hline 5 & $55387.54^{\mathrm{b}}$ & 0.49 & 230 & 21.00 & ${ }_{-0.59}^{+0.57}$ & ${ }_{-1.56}^{+2.25}$ & 306.96 & ${ }_{-1.12}^{+0.94}$ & $\begin{array}{l}+2.70 \\
-2.28\end{array}$ \\
\hline 6 & $55421.51^{\mathrm{b}}$ & 0.89 & 770 & 15.21 & $\begin{array}{l}+3.02 \\
-3.10\end{array}$ & $\begin{array}{l}+9.35 \\
-7.41\end{array}$ & 252.00 & $\begin{array}{l}+4.63 \\
-6.48\end{array}$ & $\begin{array}{l}+9.56 \\
-16.65\end{array}$ \\
\hline 7 & $55464.90^{\mathrm{b}}$ & 0.77 & 460 & 13.40 & ${ }_{-0.15}^{+0.24}$ & $\begin{array}{l}+0.52 \\
-0.45\end{array}$ & 266.29 & ${ }_{-0.23}^{+0.22}$ & ${ }_{-0.62}^{+0.58}$ \\
\hline 8 & $55478.38^{\mathrm{b}}$ & 0.86 & 660 & 11.09 & ${ }_{-0.19}^{+0.18}$ & ${ }_{-0.49}^{+0.43}$ & 331.08 & ${ }_{-0.35}^{+0.18}$ & ${ }_{-0.80}^{+0.49}$ \\
\hline 9 & $55497.30^{\mathrm{b}}$ & 0.92 & 950 & 0.50 & ${ }_{-0.10}^{+0.10}$ & ${ }_{-0.21}^{+0.25}$ & 88.95 & ${ }_{-0.25}^{+0.18}$ & ${ }_{-0.53}^{+0.48}$ \\
\hline 10 & $55513.60^{\mathrm{b}}$ & 0.80 & 520 & 3.15 & $\begin{array}{l}{ }_{-0.25}^{+0.33} \\
\end{array}$ & $\begin{array}{l}+0.70 \\
{ }_{-0.63}\end{array}$ & 285.95 & $\begin{array}{l}{ }_{-0.42}^{+0.58} \\
\end{array}$ & $\begin{array}{l}+1.29 \\
{ }_{-1.50}\end{array}$ \\
\hline 11 & $55589.56^{\mathrm{b}}$ & 0.52 & 240 & 1.03 & ${ }_{-0.08}^{+0.07}$ & $\begin{array}{l}+0.19 \\
{ }_{-0.21}\end{array}$ & 307.71 & ${ }_{-0.08}^{+0.08}$ & $\begin{array}{l}+0.52 \\
{ }_{-0.44}^{+0.52}\end{array}$ \\
\hline 12 & $55702.77^{\mathrm{b}}$ & 0.60 & 300 & 20.30 & $\begin{array}{l}-0.00 \\
{ }_{-0.62}^{+0.44}\end{array}$ & $\begin{array}{l}+1.00 \\
{ }_{-1.43}\end{array}$ & 235.13 & $\begin{array}{l}{ }_{-0.55}^{+0.89} \\
\end{array}$ & $\begin{array}{l}-2.70 \\
{ }_{-1.76}^{2}\end{array}$ \\
\hline 13 & $55722.43^{\mathrm{b}}$ & 0.47 & 210 & 35.55 & $\begin{array}{l}+0.28 \\
{ }_{-0.29}\end{array}$ & $\begin{array}{l}+0.69 \\
{ }_{-0.69}\end{array}$ & 272.22 & $\begin{array}{l}+0.50 \\
{ }_{-0.38}\end{array}$ & $\begin{array}{l}+1.23 \\
{ }_{-1.19}\end{array}$ \\
\hline 14 & $55764.22^{\mathrm{b}}$ & 0.46 & 210 & 5.29 & $\begin{array}{l}+1.87 \\
{ }_{-1.96}^{1.87}\end{array}$ & $\begin{array}{l}+4.85 \\
{ }_{-4.72}\end{array}$ & 315.66 & $\begin{array}{l}+2.37 \\
{ }_{-1.39}\end{array}$ & $\begin{array}{l}+5.31 \\
{ }_{-5} .35\end{array}$ \\
\hline 15 & $55896.86^{\mathrm{b}}$ & 0.59 & 300 & 1.87 & ${ }_{-0.37}^{+0.57}$ & $\begin{array}{l}+1.25 \\
{ }_{-1.18}\end{array}$ & 222.87 & $\begin{array}{l}+0.90 \\
{ }_{-1.14}\end{array}$ & $\begin{array}{r}+1.95 \\
{ }_{-7.73}\end{array}$ \\
\hline 16 & $55911.28^{b}$ & 0.86 & 660 & 19.10 & $\begin{array}{l}+0.54 \\
-0.77\end{array}$ & $\begin{array}{l}+2.21 \\
-2.21\end{array}$ & 36.65 & ${ }_{-0.56}^{+0.61}$ & $\begin{array}{l}{ }_{-1.71}^{+1.85} \\
\end{array}$ \\
\hline 17 & 56062.96 & 0.45 & 200 & 31.96 & ${ }_{-0.37}^{+0.30}$ & ${ }_{-0.85}^{+0.81}$ & 198.74 & ${ }_{-0.18}^{+0.49}$ & ${ }_{-1.09}^{+1.44}$ \\
\hline 18 & 56146.21 & 0.55 & 260 & 1.57 & ${ }_{-0.18}^{+0.22}$ & $\begin{array}{l}{ }_{-0.42}^{+0.46} \\
\end{array}$ & 330.10 & ${ }_{-0.36}^{+0.24}$ & $\begin{array}{l}{ }_{-0.82}^{+0.65} \\
\end{array}$ \\
\hline 19 & 56211.77 & 0.46 & 210 & -2.39 & $\begin{array}{l}{ }_{-0.19}^{+0.18} \\
\end{array}$ & ${ }_{-0.51}^{+0.42}$ & 205.11 & ${ }_{-0.24}^{+0.17}$ & $\begin{array}{l}{ }_{-0.66}^{+0.54} \\
\end{array}$ \\
\hline 20 & 56226.60 & 0.88 & 750 & 28.04 & ${ }_{-0.23}^{+0.31}$ & $\begin{array}{l}+0.67 \\
{ }_{-0.66}^{+0.67}\end{array}$ & 169.61 & $\begin{array}{l}+0.45 \\
{ }_{-0.48}^{+0.45}\end{array}$ & $\begin{array}{l}+1.16 \\
{ }_{-1.11}\end{array}$ \\
\hline 21 & $56470.11^{\mathrm{c}}$ & 0.87 & 670 & 14.46 & ${ }_{-0.39}^{+0.40}$ & $\begin{array}{l}{ }_{-0.94}^{+0.86} \\
\end{array}$ & 93.38 & ${ }_{-0.34}^{+0.33}$ & $\begin{array}{l}{ }_{-0.90}^{+.813} \\
-\end{array}$ \\
\hline 22 & 56521.83 & 0.71 & 400 & -4.44 & ${ }_{-0.39}^{+0.42}$ & $\begin{array}{l}+1.21 \\
{ }_{-0.94}\end{array}$ & 224.89 & $\begin{array}{l}+0.33 \\
{ }_{-0.32}^{+0.33}\end{array}$ & $\begin{array}{l}{ }_{-1.19}^{+0.87} \\
{ }_{-1.19}\end{array}$ \\
\hline 23 & 56579.91 & 0.49 & 390 & 10.20 & ${ }_{-0.15}^{+0.15}$ & $\begin{array}{l}+0.34 \\
{ }_{-0.49}^{+0.34}\end{array}$ & 32.94 & ${ }_{-0.27}^{+0.20}$ & $\begin{array}{l}+0.63 \\
{ }_{-0.62}\end{array}$ \\
\hline 24 & 56666.50 & 0.90 & 850 & 32.82 & ${ }_{-0.14}^{+0.16}$ & ${ }_{-0.41}^{+0.39}$ & 293.29 & ${ }_{-0.40}^{+0.18}$ & ${ }_{-1.08}^{+0.55}$ \\
\hline 25 & 56799.96 & 0.73 & 400 & 18.05 & $\begin{array}{l}+0.75 \\
{ }_{-0.63}\end{array}$ & $\begin{array}{l}+1.94 \\
{ }_{-1.80}\end{array}$ & 349.39 & ${ }_{-1.75}^{+1.13}$ & $\begin{array}{l}+2.89 \\
{ }_{-4.12}\end{array}$ \\
\hline 26 & 56817.64 & 0.66 & 340 & 1.29 & $\begin{array}{l}{ }_{-0.29}^{+0.33} \\
\end{array}$ & $\begin{array}{l}{ }_{-0.74}^{+0.83} \\
\end{array}$ & 106.26 & ${ }_{-0.74}^{+0.86}$ & $\begin{array}{l}{ }^{+2.27} \\
{ }_{-1.90}\end{array}$ \\
\hline 27 & 56819.20 & 0.995 & 4450 & 11.42 & $\begin{array}{l}{ }_{-0.08}^{+0.07} \\
\end{array}$ & ${ }_{-0.17}^{+0.17}$ & 110.63 & $\begin{array}{l}+0.16 \\
{ }_{-0.28}^{+0.16}\end{array}$ & $\begin{array}{l}+0.46 \\
{ }_{-0.55}\end{array}$ \\
\hline 28 & 57049.48 & 0.46 & 210 & 4.56 & $\begin{array}{l}{ }_{-0.12}^{+0.19} \\
\end{array}$ & $\begin{array}{l}{ }_{-0.50}^{+0.68} \\
\end{array}$ & 100.48 & $\begin{array}{l}{ }_{-0.34}^{+0.23} \\
\end{array}$ & $\begin{array}{l}{ }_{-1.87}^{+0.95} \\
\end{array}$ \\
\hline 29 & 57157.94 & 0.52 & 240 & 12.18 & $\begin{array}{l}+0.19 \\
{ }_{-0.18}\end{array}$ & $\begin{array}{l}+0.37 \\
{ }_{-0.35}\end{array}$ & 91.60 & ${ }_{-0.37}^{+0.10}$ & ${ }_{-0.74}^{+0.16}$ \\
\hline
\end{tabular}

Notes. The horizontal lines separate the different data sets IC59, IC79, IC2011 and IC2012-2014. The signalness is defined as the ratio of the astrophysical expectation over the sum of the atmospheric and astrophysical expectations for a given energy proxy and the best-fit spectrum. The signalness decreases up to about $10 \%$ when taking into account a prompt flux at the conservative upper limit of $1.06 \times$ ERS (see Section 6 ). The angular errors are statistical errors only and do not include systematics.

${ }^{a}$ These events were included in Aartsen et al. (2014c).

${ }^{b}$ These events were included in Aartsen et al. (2015c).

${ }^{c}$ This event is identical to Event 38 in Kopper et al. (2015).

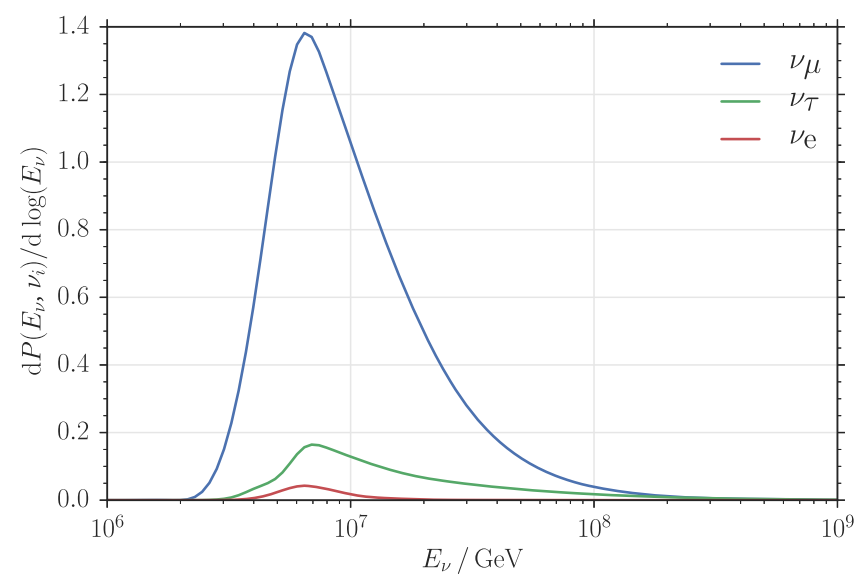

Figure 8. Probability distribution of primary neutrino energies that could result in the observed multi-PeV track-like event. The total probabilities for the different flavors are $87.7 \%, 10.9 \%$ and $1.4 \%$ for $\nu_{\mu}, \nu_{\tau}$, and $\bar{\nu}_{e}$, respectively. confirm for the Northern Hemisphere a flux of muon neutrinos that is generally consistent with the observed all flavor flux in the Southern Hemisphere, but which is in tension with the assumption of a single power law describing this and previous observations with a lower energy threshold at the same time.

It is expected that for a Galactic origin the neutrino flux should be correlated with the Galactic plane. It is generally assumed that the contribution from the Galactic plane and Galactic sources is stronger in the Southern Hemisphere, which e.g., includes the Galactic center. The measured astrophysical flux is not strongly affected by a split in right ascension (see Section 5.2), where one region includes the part of the Galactic plane which is visible in the northern sky and the other does not. This can be interpreted as an indication that the flux observed here is mostly of extragalactic origin.

The observed tension may arise either from a spectral break at lower energies for the same sources or from an additional flux component, e.g., expected from Galactic sources or the 


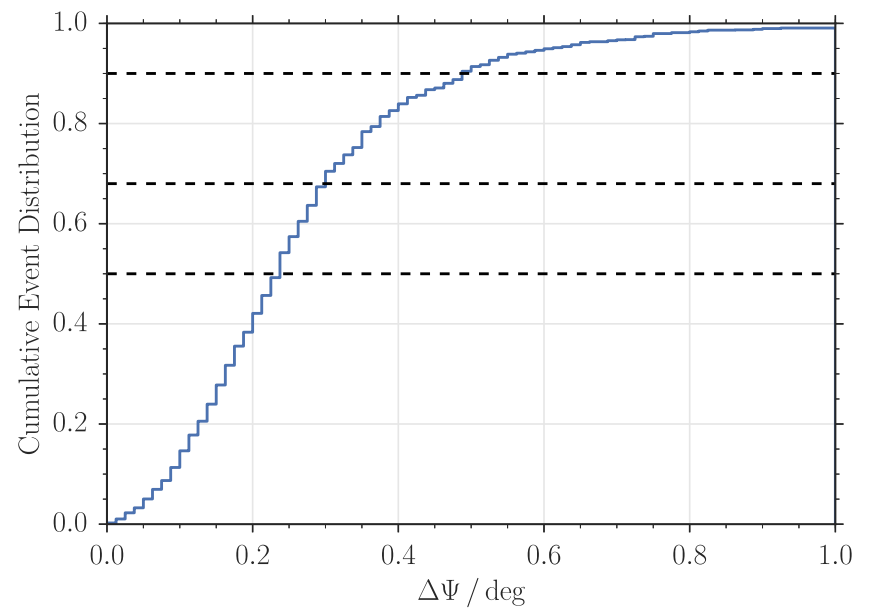

Figure 9. Angular reconstruction uncertainty of the multi-PeV track-like event. The estimate is based on an ensemble of simulated events with similar deposited energy, direction and entry point into the fiducial volume. The simulation takes into account the ice uncertainties (see Section 3.2). Including statistical and systematic uncertainties, $50 \%(99 \%)$ of the events are reconstructed better than $0.23\left(0^{\circ} .90\right)$.

Galactic plane, that is sub-dominant at the high energies to which this analysis is sensitive.

Figure 14 compares the measured diffuse astrophysical muon neutrino flux to theoretical flux predictions corresponding to different source types. The measured flux is within its uncertainties slightly below the Waxman-Bahcall upper bound (Waxman 2013). Senno et al. (2016) predict a diffuse neutrino flux originating from gamma-ray bursts, which is currently not ruled out (Aartsen et al. 2015d, 2016). A flux of cosmogenic neutrinos as predicted by Kotera et al. (2010) would only contribute subdominantly to the measured astrophysical neutrino flux. Neutrino fluxes from blazars and star-forming galaxies are predicted by e.g., Murase et al. (2014) and Bechtol et al. (2015), respectively. Glüsenkamp (2016) already constrains this blazar model. These fluxes are of the same order of magnitude as the measured flux within the given uncertainty band. However, due to the small statistics at high energies we cannot differentiate if the measured astrophysical neutrino flux corresponds to a neutrino flux originating from a specific source type or if it is a combination of different source types.

\section{ANALYSIS OF ARRIVAL DIRECTIONS AND SEARCH FOR ANISOTROPIES}

\subsection{Arrival Directions of Highest-energy Events}

The multi-PeV event discussed in Section 4.3 has a high probability of being astrophysical. Therefore, it is particularly interesting to correlate such an event with potential sources.

Figure 15 shows the direction of the event with its angular uncertainty and nearby high-energy gamma-ray sources from Nolan et al. (2012), Acero et al. (2015), Wakely \& Horan (2007) in a window centered around the arrival direction. The closest source is multiple degrees away which is much larger than the angular error estimate.

For events that have a muon energy proxy above $200 \mathrm{TeV}$ we expect roughly twice as many events with an astrophysical origin than with an atmospheric origin, assuming the best-fit
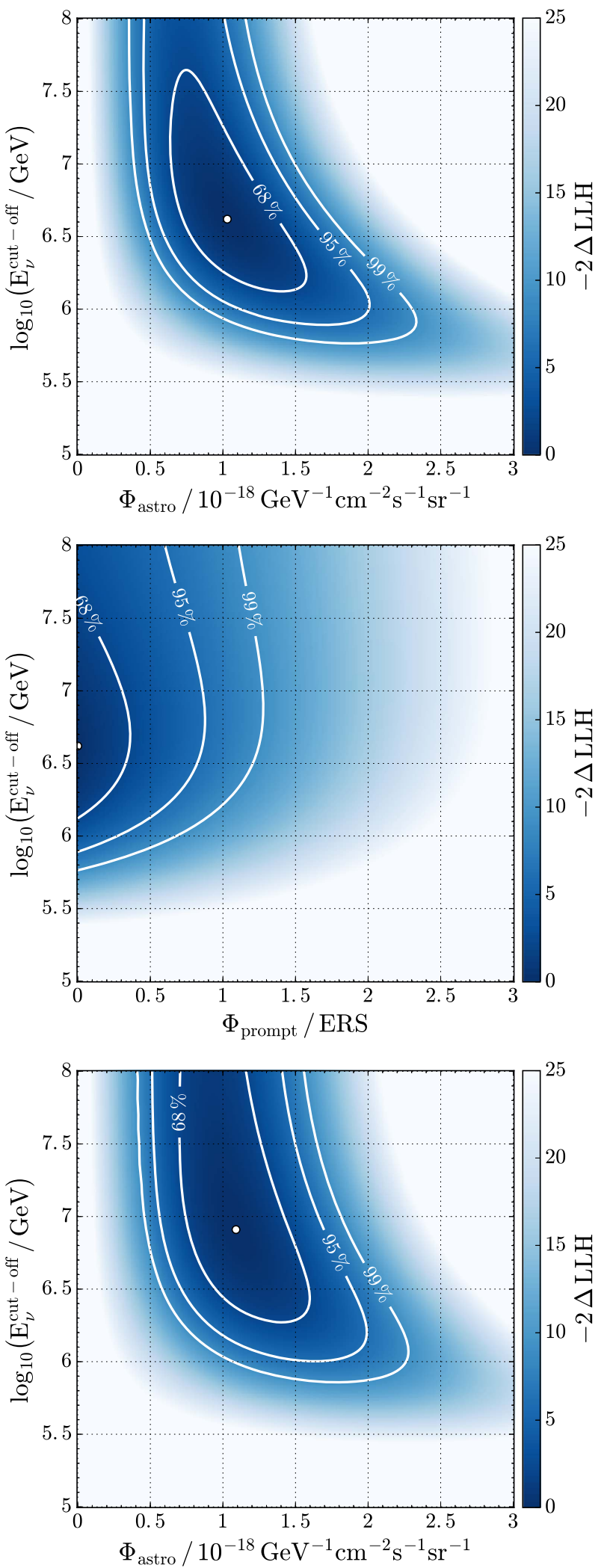

Figure 10. Two-dimensional profile likelihood scans of the astrophysical parameters $\Phi_{\text {astro }}, E_{\nu}^{\text {cut-off }}$ and the prompt normalization $\Phi_{\text {prompt }}$ in units of the model in Enberg et al. (2008). The contour lines at 68\%, 90\% and $95 \% \mathrm{CL}$ assume Wilks' theorem. For the top and middle figure the spectral index is fixed to $\gamma_{\text {astro }}=2$, while in the bottom figure it is fixed to the best-fit value $\gamma_{\text {astro }}=2.13$. The white dots indicate the best-fit values. 


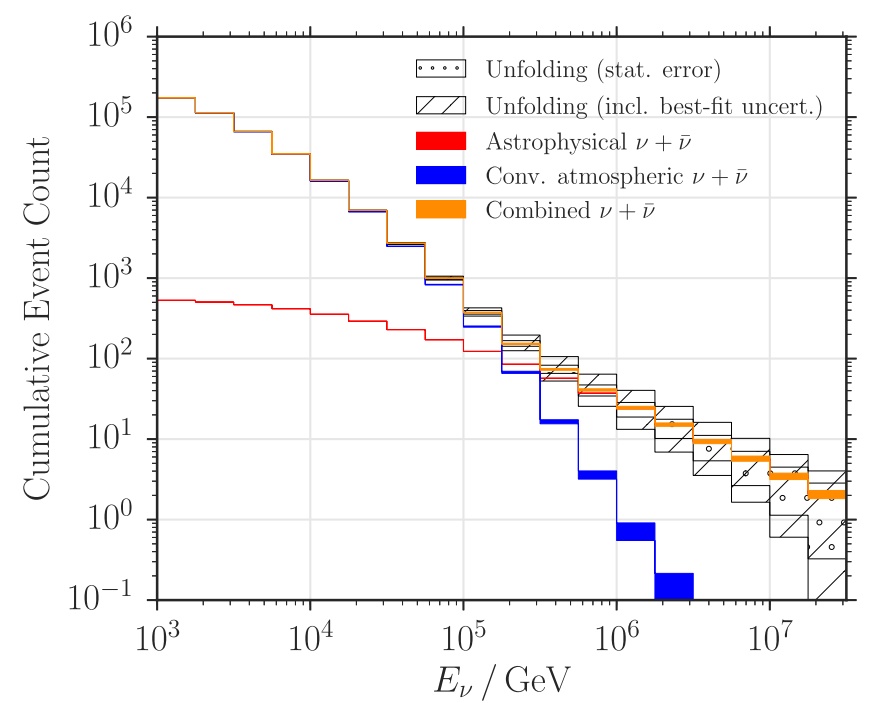

Figure 11. Cumulative distribution of the parametric unfolded neutrino energy spectrum for the six-year data sample assuming the best-fit spectrum as given by this analysis. Blue/red corresponds to the conventional atmospheric/ astrophysical expectation weighted to the best-fit spectrum. Orange represents the sum of both expectations. The parametric unfolded data are shown as a hatched band where the gray band shows the statistical uncertainty and the white band additionally the effect of the uncertainties on the fitted astrophysical flux parameters.

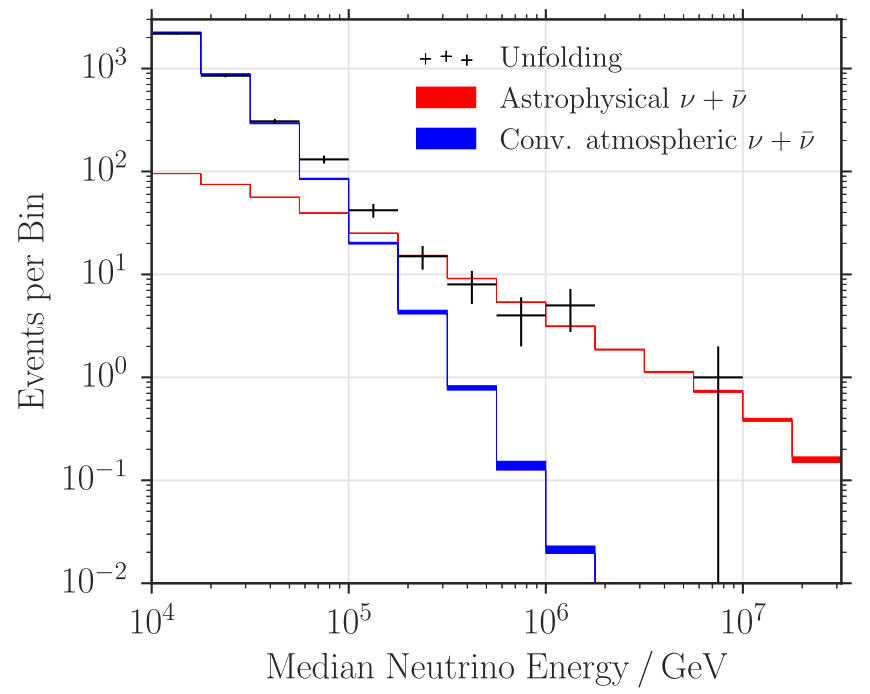

Figure 12. Distribution of the median expected neutrino energy assuming the best-fit spectrum as given by this analysis. The black crosses corresponds to experimental data and blue/red to the conventional atmospheric/astrophysical expectation weighted to the best-fit spectrum.

spectrum. Figure 16 shows the arrival direction of these events. Most events are located relatively close to the horizon where the Earth is not yet opaque to high energy neutrinos. Table 4 summarizes the per-event information. No obvious correlation with gamma-ray sources in the catalogs of Nolan et al. (2012), Acero et al. (2015), or Wakely \& Horan (2007) were found. However, event 10 is close to the extended TeV source HESS J1857+026 (Wakely \& Horan 2007).

A dedicated analysis searching for clusters in the neutrino arrival directions has been performed and found no evidence for a neutrino point source (M. G. Aartsen et al. 2016, in preparation).

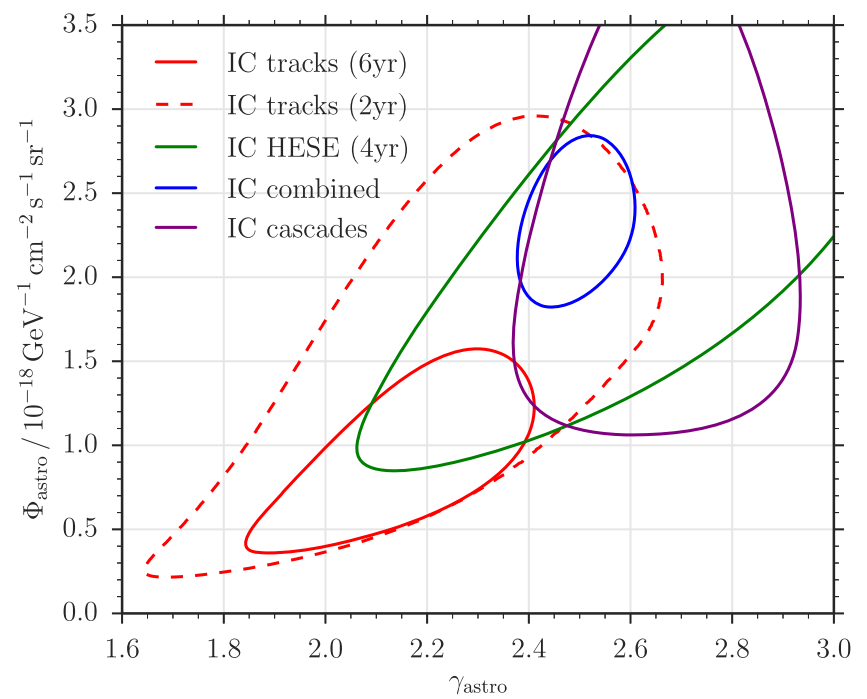

Figure 13. Results of different IceCube analyses measuring the astrophysical flux parameters $\Phi_{\text {astro }}$ and $\gamma_{\text {astro }}$. The contour lines show the $90 \% \mathrm{CL}$. The result of this analysis (IC tracks, 6yr) is shown by the red solid contour line. The contour obtained by the previous measurement using through-going muons (Aartsen et al. 2015c) (IC tracks, 2yr) is the red dashed line. In addition, the results for the most recent analysis of starting events (Kopper et al. 2015) (IC HESE, 4yr), the complementary cascade channel (Niederhausen et al. 2015) (IC cascades) and an analysis combining different IceCube results (IC combined, Aartsen et al. 2015f) are shown. The result of this analysis (red, solid) and IC combined are incompatible at $3.3 \sigma$ (two-sided significance).

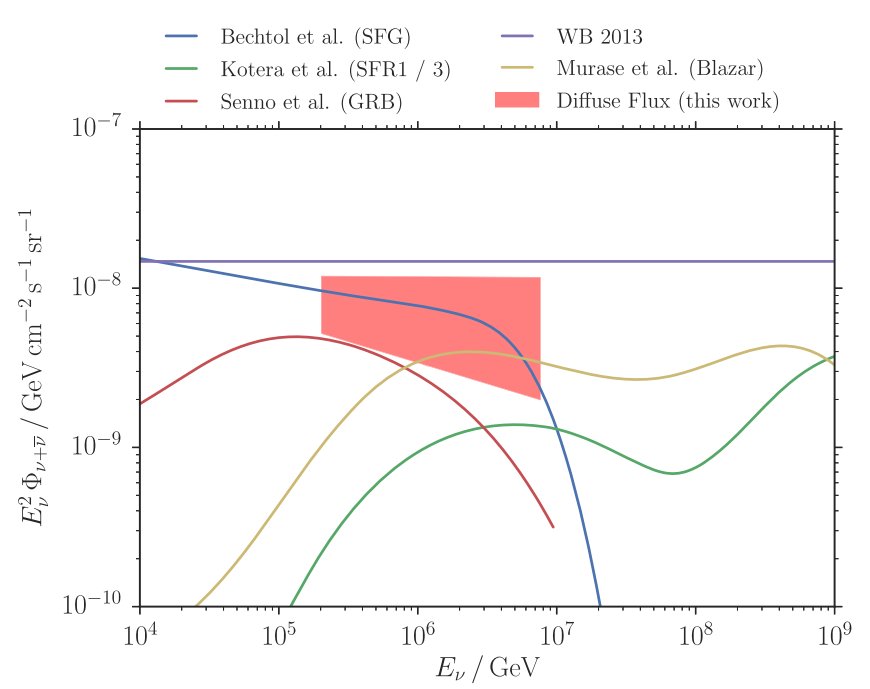

Figure 14. Comparison of the measured diffuse astrophysical muon neutrino flux (see Figure 5) with theoretical neutrino flux predictions corresponding to different source types (Kotera et al. 2010; Murase et al. 2014; Bechtol et al. 2015; Senno et al. 2016). Since Murase et al. 2014 predicts a lower and upper flux bound for neutrinos originating from blazars the central line between both bounds is shown. The purple line shows the Waxman-Bahcall upper bound (Waxman 2013).

\subsection{Test for Anisotropies Related to the Galactic Plane}

As discussed in Section 4.6 the measurement in this paper confirms the observation of an all-sky diffuse high-energy astrophysical neutrino flux. However, a tension exists between the measured spectral index of this analysis with the starting event data which originate mostly from the Southern Hemisphere. Furthermore, Neronov \& Semikoz (2016) claim inconsistency of the previously published starting event data 


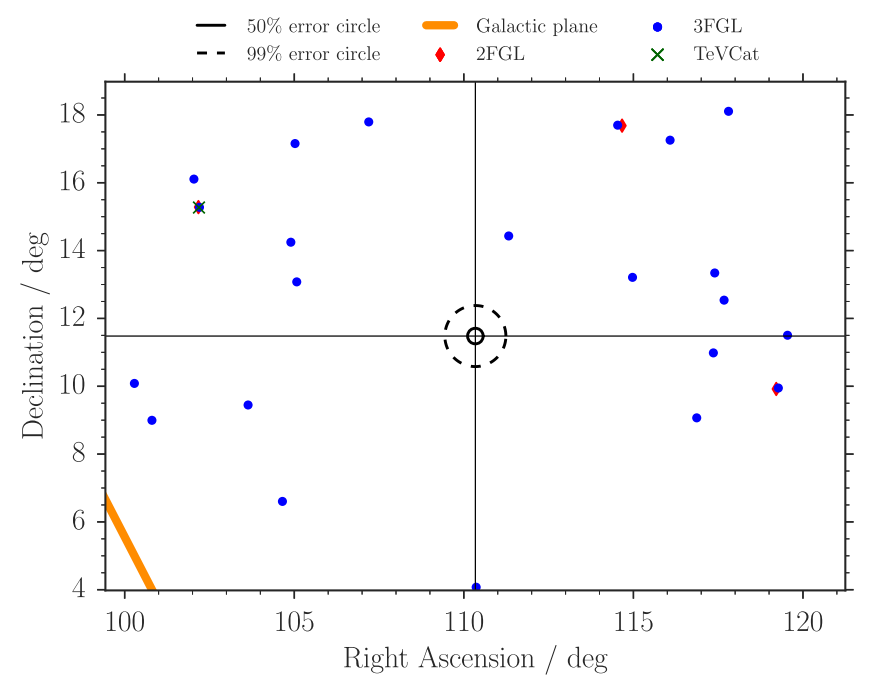

Figure 15. Window centered around the arrival direction of the multi-PeV track-like event. The solid (dashed) black line shows the 50\% (99\%) error circle for the angular reconstruction. The orange line indicates the Galactic plane. Additionally, the gamma-ray sources of the catalogs Wakely \& Horan (2007), Acero et al. (2015), Nolan et al. (2012) within the window are shown.

with an isotropic signal with a preference of a Galactic latitude dependency. As the comparison to the Southern Hemisphere is subject to different energy thresholds and detector systematics, we perform a simple, self-consistent test for a dominant signal from the Galactic plane.

We split the sample into two right ascension regions, one containing the main parts of the Galactic plane: $\alpha \in\left[0.0,108^{\circ} .9\right) \cup\left[275^{\circ} .0,360^{\circ} .0\right)$ and one excluding it: $\alpha \in\left[108^{\circ} .9,275^{\circ} .0\right)$. These intervals are chosen such that the two split samples are of similar statistics, resulting in 162363 and 189931 events respectively. Both samples are fitted independently and the aforementioned systematics can be considered identical as they are equalized by the daily Earth rotation.

The fit result, shown in Figure 17, is a small but not statistically significant larger flux and softer spectrum from the region including the Galactic plane. The $p$-value for both results being compatible is at about $43 \%$. In conclusion, the observed flux is not dominated by the Galactic plane. However a small, sub-dominant contribution cannot be excluded.

\section{SEARCH FOR A SIGNATURE OF PROMPT ATMOSPHERIC NEUTRINOS}

The expected prompt neutrino flux provides a background for the measurement of the astrophysical flux. However, a flux of prompt neutrinos is interesting by itself and can be constrained by the present analysis.

The prompt flux predicted by Enberg et al. (2008) is subdominant to the conventional flux at low energies and the astrophysical flux at high energies. Nevertheless, the correlation of the energy spectrum and arrival directions of neutrinos at the detector lead to a clear signature. Figure 18 shows the pulls for simulated data corresponding to six years of live time and based on the IC2012-2014 event selection. Here, signal is defined as the prompt expectation and background is the sum of the conventional and astrophysical flux. The main effect of a prompt neutrino flux on the two observables will be visible for muon energy proxy values between 1 and $100 \mathrm{TeV}$ in the fairly up-going directions. However, a large part of this signature is absorbed within the uncertainties represented by the implemented nuisance parameters (see Section 3.2).

The overall best-fit prompt normalization is zero. Figure 19 shows the best-fit prompt normalization as a function of the astrophysical normalization and spectral index. Additionally, the two-dimensional confidence contours for the astrophysical parameters are shown. In the region where our experimental data are compatible with our single power-law model, the bestfit prompt normalization does not deviate from zero. Only for strong deviations from the best-fit astrophysical spectrum is a non-zero prompt normalization fitted, but this is strongly disfavored with respect to the best fit. Such behavior is expected. If the astrophysical flux decreases, the measured high-energy events need to be explained by another component. Assuming an unbroken power-law model for the astrophysical flux, the sensitivity for the prompt neutrino flux, taking into account the systematic uncertainties, is estimated to be $1.5 \times$ ERS. Note that the sensitivity (median expected upper limit in the absence of a prompt neutrino flux) on a prompt neutrino flux depends on the chosen input values for the astrophysical flux.

In the absence of an indication of a non-zero prompt contribution an upper limit is calculated. Based on the profile likelihood for the prompt normalization, the upper limit at $90 \%$ confidence level is $0.50 \times \mathrm{ERS}$. The more stringent limit compared to the sensitivity is caused by an under-fluctuation of the conventional atmospheric and astrophysical background by about one standard deviation.

For this reason we scan the resulting limit on the prompt flux as a function of the astrophysical signal parameters.

Figure 20 shows the joint three-dimensional 90\% confidence region for the prompt flux and the astrophysical parameters. It was obtained using Wilks' theorem, and is bound by the surface for which $-2 \Delta \log L$ is 6.25 higher than the best-fit value. The maximum prompt flux in the three-dimensional confidence region is $1.06 \times \mathrm{ERS}$. We take this as a conservative upper limit on the prompt flux. Further tests have shown that reasonable changes to the astrophysical hypothesis, such as the introduction of a high-energy cut-off, have only small effects on this limit.

Several more recent calculations of the prompt flux have been published: GMS (H3p) (Garzelli et al. 2015), BERSS (H3p) (Bhattacharya et al. 2015) and GRRST (H3p) (Gauld et al. 2016). Figure 21 shows multiple predictions for the prompt flux as well as the upper limit calculated here using the prediction from Enberg et al. (2008) and taking into account a more realistic cosmic-ray model (Gaisser 2012). Since nuisance parameters describing the uncertainties of the cosmic-ray model, e.g., the cosmic-ray spectral index, are implemented, the upper limit curve slightly deviates from the ERS prediction including the knee. The energy range has been calculated such that the limit increases by $10 \%$ if only neutrinos with energies in that range are taken into account. For the sensitive region which is between 9 and $69 \mathrm{TeV}$ the effect of the prompt predictions is only a change in normalization and it is therefore appropriate to convert the limit obtained with the ERS prediction to the other predictions. Also, the cosmic-ray composition only changes the normalization in this energy range. The values are summarized in Table 5 . 


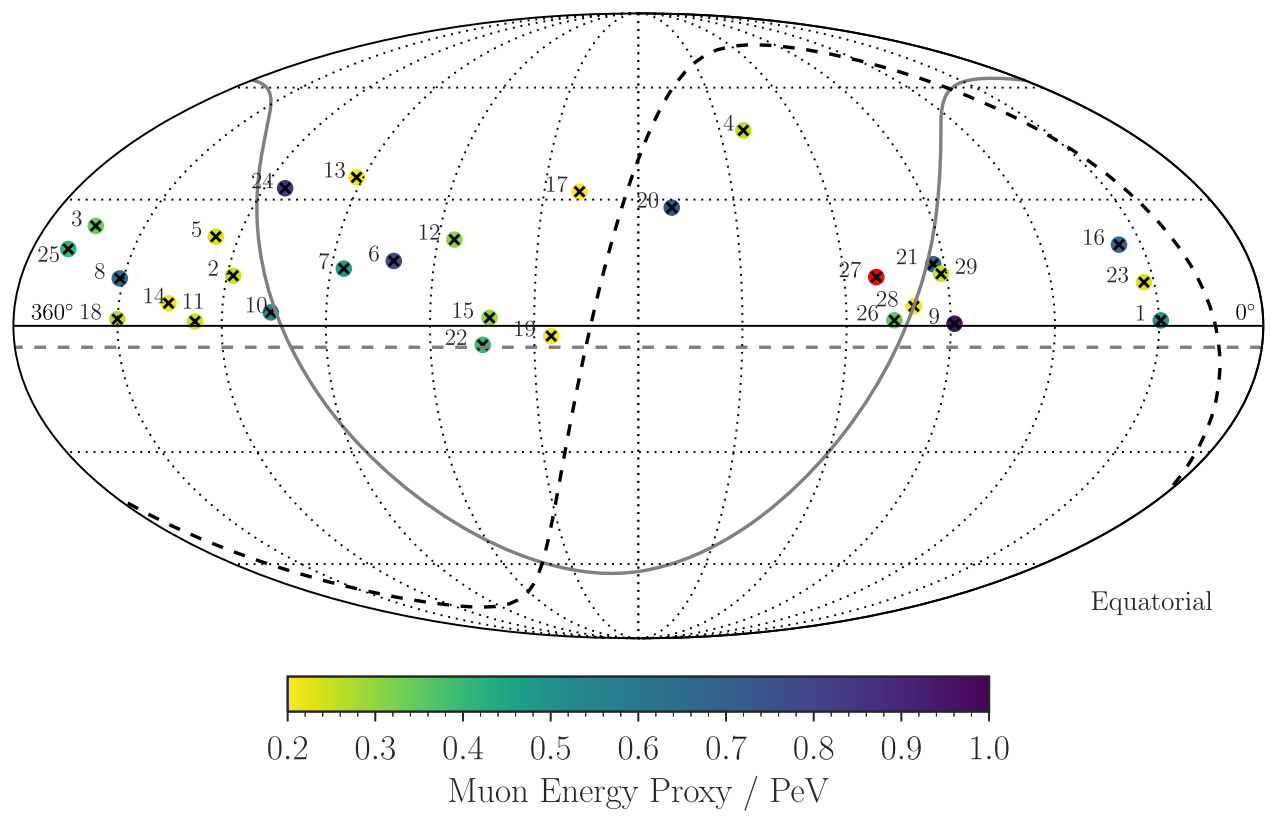

Figure 16. Arrival directions of events with a muon energy proxy above $200 \mathrm{TeV}$. Given the best-fit spectrum the ratio of astrophysical to atmospheric events is about two to one. The horizontal dashed gray line shows the applied zenith angle cut of $85^{\circ}$. The curved gray line indicates the Galactic plane and the dashed black line the supergalactic plane (Lahav et al. 2000). The multi-PeV track event is shown as a red dot and the energy proxy value listed in Table 4.

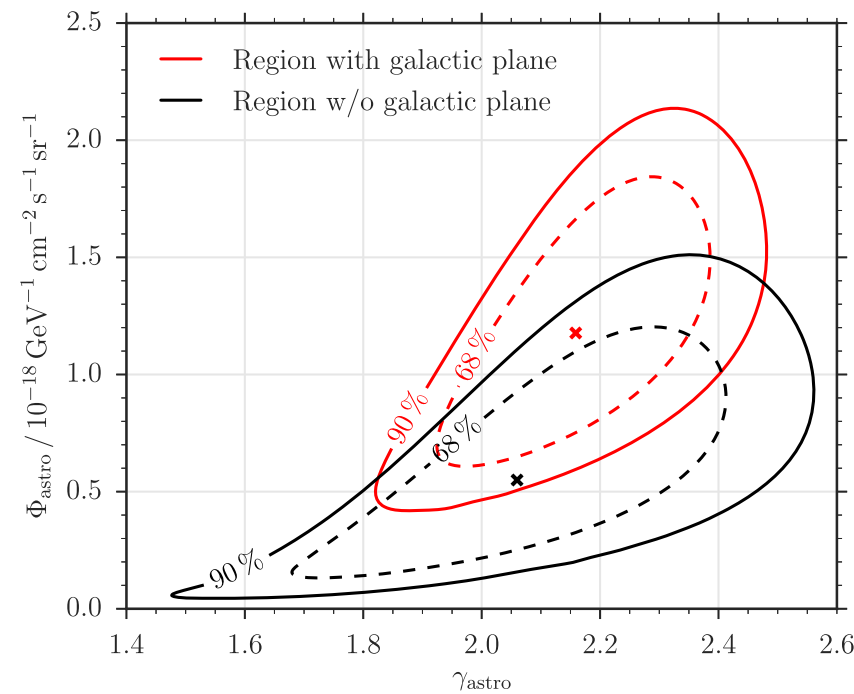

Figure 17. Two-dimensional profile likelihood scans of the astrophysical parameters $\Phi_{\text {astro }}$ and $\gamma_{\text {astro }}$ for the two disjoint right ascension regions, one containing the Northern Hemisphere part of the Galactic plane (red) and the other not (black). The contour lines at 68\% and 90\% CL assume Wilks' theorem.

\section{SUMMARY AND CONCLUSIONS}

In this paper we have presented the result of analyzing six years of up-going muon data measured with the IceCube neutrino telescope. We measure an astrophysical flux of $\Phi_{\nu+\bar{\nu}}=\left(0.90_{-0.27}^{+0.30}\right) 10^{-18} \mathrm{GeV}^{-1} \mathrm{~cm}^{-2} \mathrm{sr}^{-1} \mathrm{~s}^{-1}$.

$\left(E_{\nu} / 100 \mathrm{TeV}\right)^{-(2.13 \pm 0.13)}$ with a statistical significance of 5.6 standard deviations with respect to only being of atmospheric origin. With this result we have further established the observation of an astrophysical neutrino signal (Aartsen et al. 2013a, 2014b, 2015c) in a second, largely independent detection channel. The detection channel used here is of great interest because of the good directional reconstruction of

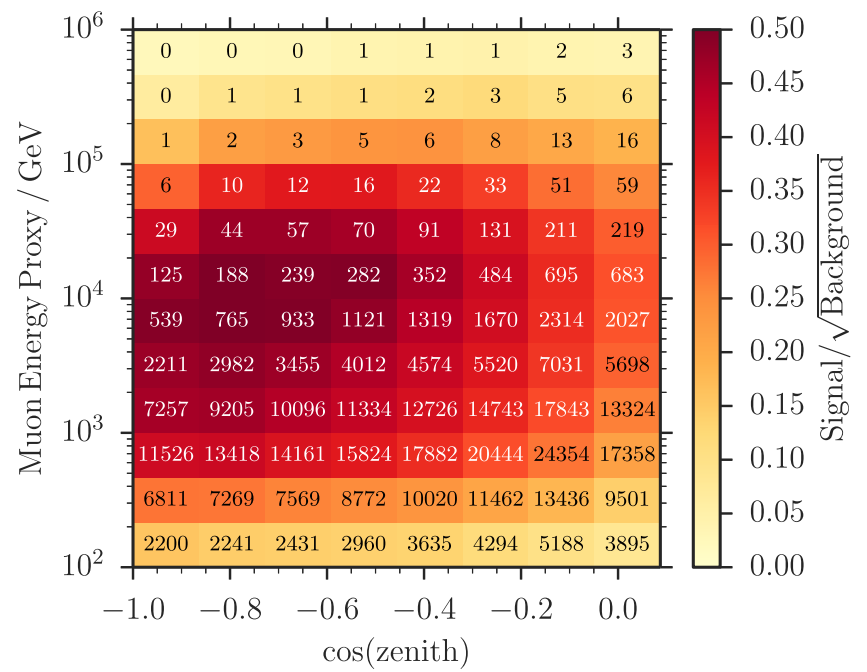

Figure 18. Signal over square root of background for the reconstructed muon energy vs. zenith angle corresponding to six years of IceCube data after applying the event selection for the 86-string configuration (IC2012-2014). Here, background is defined as the sum of the conventional atmospheric (Honda et al. 2007) and astrophysical $\left(10^{-8} \times E^{-2}\right) \nu_{\mu}+\bar{\nu}_{\mu}$ flux. The prompt atmospheric (Enberg et al. 2008) $\nu_{\mu}+\bar{\nu}_{\mu}$ flux is defined as the signal. The numbers in each bin correspond to the expected number of background events in six years.

detected muons and a large signal efficiency with an estimated number of about 500 astrophysical neutrinos included in this data sample.

The data include an exceptionally high-energy muon with $(2.6 \pm 0.3) \backslash \mathrm{PeV}$ deposited energy, which is the highest-energy lepton that has been reported to date.

A parametric unfolding of neutrino energies shows that the spectrum extends to about $10 \mathrm{PeV}$ in neutrino energy with no significant spectral break or cut-off.

The measured hard spectral index of $\gamma=2.13 \pm 0.13$ is in tension with complementary measurements of IceCube, which 


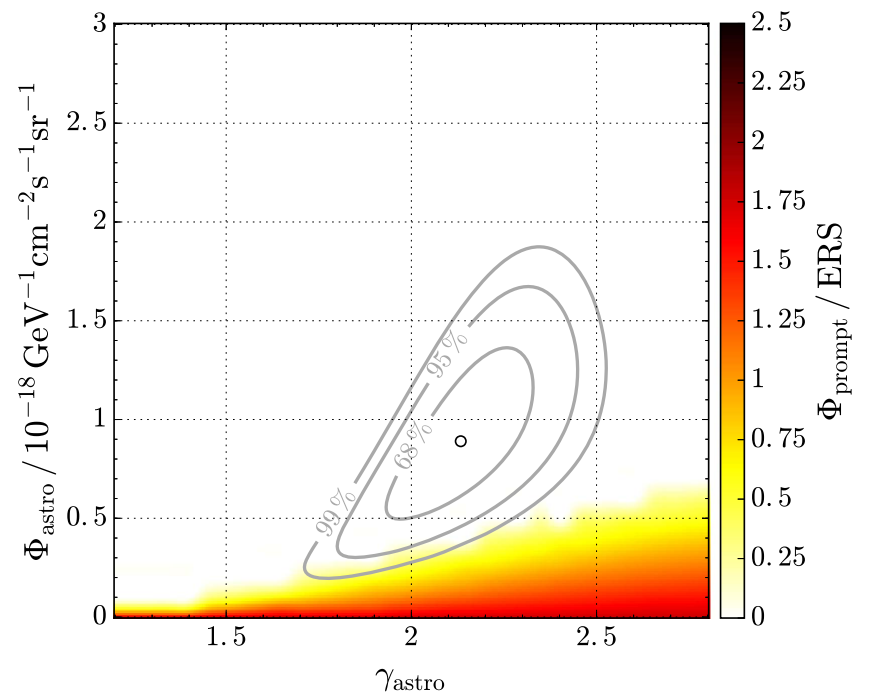

Figure 19. Best-fit prompt normalization $\Phi_{\text {prompt }}$ in units of the model of Enberg et al. (2008) for each scan point $\Phi_{\text {astro }}, \gamma_{\text {astro }}$. Additionally, the twodimensional contours for $\Phi_{\text {astro }}, \gamma_{\text {astro }}$ are shown.

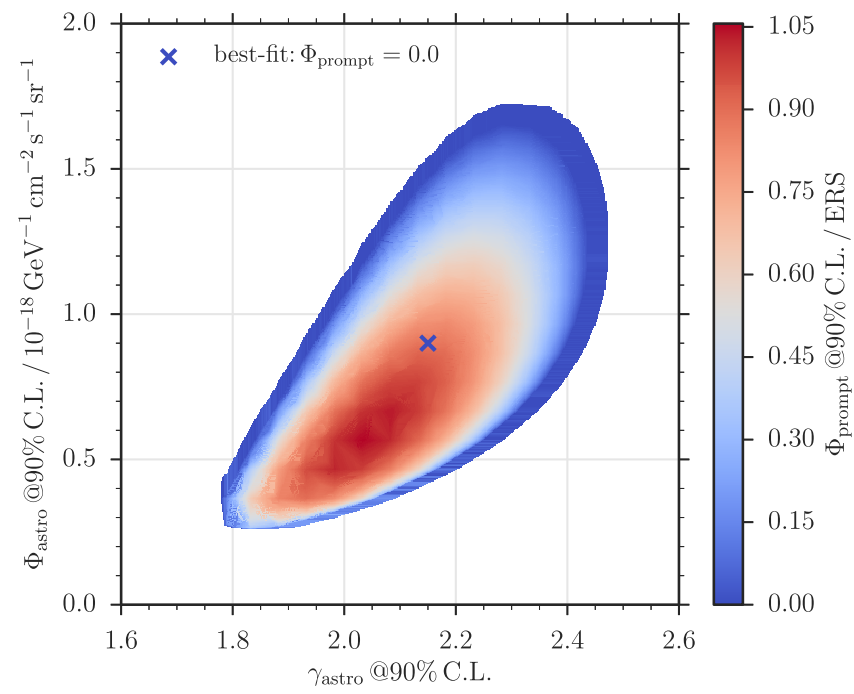

Figure 20. $90 \%$ CL contour assuming Wilks' theorem based on a threedimensional profile likelihood scans of the astrophysical parameters $\Phi_{\text {astro }}, \gamma_{\text {astro }}$ and the prompt normalization $\Phi_{\text {prompt }}$ in units of the model of Enberg et al. (2008).

have a lower energy threshold by about one order of magnitude and are predominantly sensitive to the Southern Hemisphere. However, the consistency of the observed fluxes at high energies may be interpreted as indication of a spectral break or additional astrophysical component at lower energy to which this analysis is not sensitive.

For the highest-energy events no correlation with known high-energy gamma-ray sources or other astrophysical objects could be identified.

By splitting the data in right ascension, we find no significant correlation with the orientation of the Galactic plane and conclude that the dominant fraction of the flux is largely all-sky and isotropic.

The present analysis is also sensitive to a flux of prompt neutrinos which are expected from the decay of heavy mesons in the atmosphere. We find no indications for such a signal. However, because the prompt flux is subdominant to the

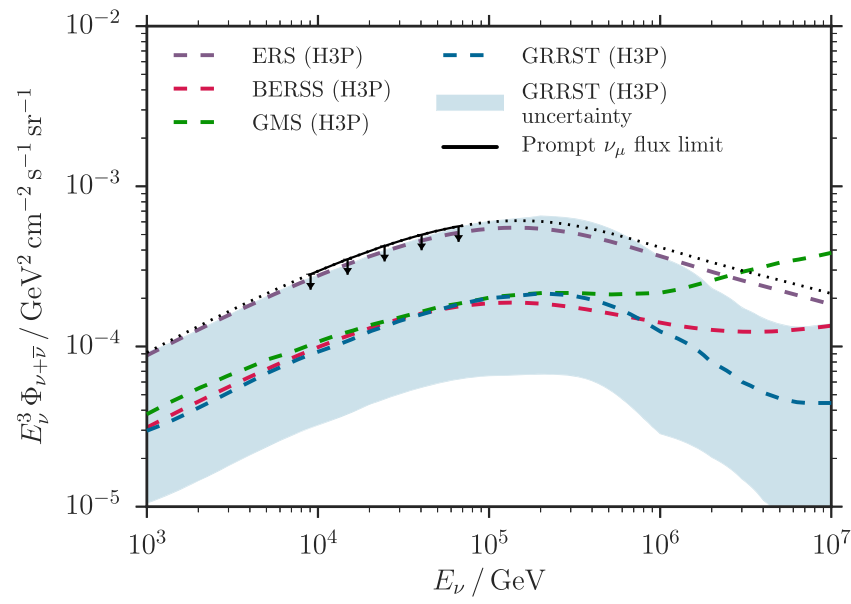

Figure 21. Prompt atmospheric muon neutrino flux predictions shown as dashed lines (Enberg et al. 2008; Bhattacharya et al. 2015; Garzelli et al. 2015; Gauld et al. 2016) in comparison to the constraint on the prompt flux given by this analysis. The shaded area shows the uncertainty band corresponding to the prediction in Gauld et al. (2016). Besides the ERS (H3p) predicition this is the closest band to the prompt flux constraint. For better readability the uncertainty bands of the other models are not shown. The black solid line shows the neutrino energy region where the prompt neutrino flux based on the model in Enberg et al. (2008) is constrained. The black dotted line indicates the model behavior including the best-fit nuisance parameters beyond the sensitive energy range. All flux predictions are based on the cosmic-ray model from Gaisser (2012).

Table 5

Limits for Fluxes of Prompt Neutrinos for Different Predictions

\begin{tabular}{lc}
\hline \hline Model & Flux limit \\
\hline ERS (H3p) & 1.06 \\
GMS (H3p) & $\approx 2.9$ \\
BERSS (H3p) & $\approx 3.0$ \\
GRRST (H3p) & $\approx 3.1$ \\
\hline
\end{tabular}

Note. The limits for GMS (H3p) (Garzelli et al. 2015), BERSS (H3p) (Bhattacharya et al. 2015) and GRRST (H3p) (Gauld et al. 2016) are determined by rescaling the ERS (H3p) limit with the corresponding flux ratio at $30 \mathrm{TeV}$ which is well within the sensitive energy range. All flux predictions are based on the cosmic ray model from Gaisser (2012).

astrophysical and conventional atmospheric neutrino flux, the exclusion depends on the assumed astrophysical model parameters. Variations of the astrophysical flux uncertainties lead to a conservative exclusion limit of approximately at the level of the mean expected flux normalization from Enberg et al. (2008). For the first time, it is possible to constrain such a flux in this range of theoretical predictions. However, recent perturbative QCD calculations from Garzelli et al. (2015), Bhattacharya et al. (2015) and (Gauld et al. 2016) predict lower prompt neutrino fluxes which are not yet constrained by the upper limit.

We acknowledge the support from the following agencies: U.S. National Science Foundation-Office of Polar Programs, U.S. National Science Foundation-Physics Division, University of Wisconsin Alumni Research Foundation, the Grid Laboratory Of Wisconsin (GLOW) grid infrastructure at the University of Wisconsin-Madison, the Open Science Grid (OSG) grid infrastructure; U.S. Department of Energy, and 
National Energy Research Scientific Computing Center, the Louisiana Optical Network Initiative (LONI) grid computing resources; Natural Sciences and Engineering Research Council of Canada, WestGrid and Compute/Calcul Canada; Swedish Research Council, Swedish Polar Research Secretariat, Swedish National Infrastructure for Computing (SNIC), and Knut and Alice Wallenberg Foundation, Sweden; German Ministry for Education and Research (BMBF), Deutsche Forschungsgemeinschaft (DFG), Helmholtz Alliance for Astroparticle Physics (HAP), Research Department of Plasmas with Complex Interactions (Bochum), Germany; Fund for Scientific Research (FNRS-FWO), FWO Odysseus programme, Flanders Institute to encourage scientific and technological research in industry (IWT), Belgian Federal Science Policy Office (Belspo); University of Oxford, United Kingdom; Marsden Fund, New Zealand; Australian Research Council; Japan Society for Promotion of Science (JSPS); the Swiss National Science Foundation (SNSF), Switzerland; National Research Foundation of Korea (NRF); and Villum Fonden, Danish National Research Foundation (DNRF), Denmark.

\section{REFERENCES}

Aartsen, M. G., Abbasi, R., Abdou, Y., Ackermann, M., \& Adams, J. 2013a, Sci, 342, 1242856

Aartsen, M. G., Abbasi, R., Abdou, Y., et al. 2013b, NIMPA, 711, 73

Aartsen, M. G., Abbasi, R., Ackermann, M., et al. 2014a, JInst, 9, P03009

Aartsen, M. G., Abbasi, R., Ackermann, M., et al. 2014c, PhRvD, 89, 062007

Aartsen, M. G., Abraham, K., Ackermann, M., et al. 2015a, ApJ, 809, 98

Aartsen, M. G., Abraham, K., Ackermann, M., et al. 2015f, in Proc. 34th ICRC, 21

Aartsen, M. G., Abraham, K., Ackermann, M., et al. 2015c, PhRvL, 115, 081102

Aartsen, M. G., Abraham, K., Ackermann, M., et al. 2016, ApJ, submitted (arXiv: 1601.06484)

Aartsen, M. G., Ackermann, M., Adams, J., et al. 2014b, PhRvL, 113, 101101

Aartsen, M. G., Ackermann, M., Adams, J., et al. 2015b, PhRvD, 91, 022001

Aartsen, M. G., Ackermann, M., Adams, J., et al. 2015d, ApJL, 805, L5

Aartsen, M. G., Ackermann, M., Adams, J., et al. 2015e, ApJ, 807, 46

Abbasi, R., Abdou, Y., Abu-Zayyad, T., et al. 2010, NIMPA, 618, 139

Abbasi, R., Abdou, Y., Ackermann, M., et al. 2013, NIMPA, 703, 190

Abbasi, R., Ackermann, M., Adams, J., et al. 2009, NIMPA, 601, 294

Acero, F., Ackermann, M., Ajello, M., et al. 2015, ApJS, in press (arXiv:1501. 02003)

Achterberg, A., Ackermann, M., Adams, J., et al. 2006, APh, 26, 155

Ackermann, M., Ahrens, J., Bai, X., et al. 2006, JGRD, 111, D13

Ageron, M., Aguilar, J. A., Al Samarai, I., et al. 2011, NIMPA, 656, 11

Ahrens, J., Bai, X., Bay, R., et al. 2004, NIMPA, 524, 169

AIRS Science Team/Joao Texeira, 2013, Aqua AIRS Level 3 Daily Standard Physical Retrieval (AIRS+AMSU), version 006, Tech. Rep. (Washington, DC: NASA)

Anchordoqui, L. A., Goldberg, H., Lynch, M. H., et al. 2014, PhRvD, 89, 083003

Andres, E., Askebjer, P., Barwick, S., et al. 2000, APh, 13, 1

Athar, H., Kim, C. S., \& Lee, J. 2006, MPLA, 21, 1049

Bechtol, K., Ahlers, M., Di Mauro, M., Ajello, M., \& Vandenbroucke, J. 2015, ApJ, submitted (arXiv:1511.00688)

Becker, J. K. 2008, PhR, 458, 173

Bell, A. 2013, APh, 43, 56

Belolaptikov, I. A., Bezrukov, L. B., Borisovets, B. A., et al. 1997, APh, 7, 263

Bhattacharya, A., Enberg, R., Reno, M. H., Sarcevic, I., \& Stasto, A. 2015, JHEP, 2015, 110

Boerner, M., Ruhe, T., Scheriau, F., \& Schmitz, M. 2015, in Proc. 34th ICRC, 53
Chirkin, D. 2013a, in Proc. 33rd ICRC, 17

Chirkin, D. 2013b, arXiv:1304.0735

Chirkin, D. 2013c, NIMPA, 725, 141

Chirkin, D., \& Rhode, W. 2004, arXiv:hep-ph/0407075

Cholis, I., \& Hooper, D. 2013, JCAP, 2013, 030

Cooper-Sarkar, A., Mertsch, P., \& Sarkar, S. 2011, JHEP, 08, 042

Desiati, P., et al. 2014, in Proc. 33rd ICRC, 0492

Enberg, R., Reno, M. H., \& Sarcevic, I. 2008, PhRvD, 78, 043005

Freund, Y., \& Schapire, R. E. 1997, J. Comput. Syst. Sci., 55, 119

Gaisser, T. K. 1990, Cosmic Rays and Particle Physics (Cambridge: Cambridge Univ. Press)

Gaisser, T. K. 2012, APh, 35, 801

Gaisser, T. K., Halzen, F., \& Stanev, T. 1995, PhR, 258, 173

Garzelli, M. V., Moch, S., \& Sigl, G. 2015, JHEP, 2015, 115

Gauld, R., Rojo, J., Rottoli, L., Sarkar, S., \& Talbert, J. 2016, JHEP, 02,130

Gazizov, A., \& Kowalski, M. P. 2005, CoPhC, 172, 203

Glüsenkamp, T. 2016, EPJ, 121, 05006

Gonzalez-Garcia, M., Halzen, F., \& Niro, V. 2014, APh, 57-58, 39

Greisen, K. 1960, ARNPS, 10, 63

He, H.-N., Wang, T., Fan, Y.-Z., Liu, S.-M., \& Wei, D.-M. 2013, PhRvD, 87, 063011

Heck, D., Schatz, G., Thouw, T., Knapp, J., \& Capdevielle, J. 1998, CORSIKA: A Monte Carlo Code to Simulate Extensive Air Showers, Tech. Rep. (Karlsruhe: KIT)

Hoerandel, J. R. 2003, APh, 19, 193

Honda, M., Kajita, T., Kasahara, K., Midorikawa, S., \& Sanuki, T. 2007, PhRvD, 75, 043006

Illana, J. I., Lipari, P., Masip, M., \& Meloni, D. 2011, APh, 34, 663

Kalashev, O. E., Kusenko, A., \& Essey, W. 2013, PhRvL, 111, 041103

Kashti, T., \& Waxman, E. 2005, PhRvL, 95, 181101

Klein, S. R., Mikkelsen, R. E., \& Becker Tjus, J. 2013, ApJ, 779, 106

Koehne, J. H., Frantzen, K., Schmitz, M., et al. 2013, CoPhC, 184, 2070

Kopper, C., Kurahashi, N., et al. 2015, in Proc. 34th ICRC, 45

Kotera, K., Allard, D., \& Olinto, A. V. 2010, JCAP, 1010, 013

Laha, R., Beacom, J. F., Dasgupta, B., Horiuchi, S., \& Murase, K. 2013, PhRvD, 88, 043009

Lahav, O., Santiago, B., Webster, A., et al. 2000, MNRAS, 312, 166

Lai, H. L., Huston, J., Kuhlmann, S., et al. 2000, EPJC, 12, 375

Learned, J., \& Mannheim, K. 2000, ARNPS, 50, 679

Learned, J. G., \& Pakvasa, S. 1995, APh, 3, 267

Lundberg, J., Miocinovic, P., Burgess, T., et al. 2007, NIMPA, 581,619

Markov, M. 1960, in Proc. 10th Int. Conf. on High-Energy Physics, ed. E. C. G. Sudarshan, J. H. Tinlot, \& A. C. Melissinos (Rochester, NY: Rochester Univ. Press), 578

Murase, K., Inoue, Y., \& Dermer, C. D. 2014, PhRvD, 90, 023007

Narsky, I., \& Porter, F. C. 2013, Statistical Analysis Techniques in Particle Physics: Fits, Density Estimation and Supervised Learning (New York: Wiley)

Neronov, A., \& Semikoz, D. V. 2016, APh, 75, 60

Niederhausen, H., Lesiak-Bzdak, M., Stössl, A., et al. 2015, in Proc. 34th ICRC, 59

Nolan, P. L., Abdo, A. A., Ackermann, M., et al. 2012, ApJS, 199, 31

Olive, K. A., et al. 2014, ChPhC, 38, 090001

Pedregosa, F., Varoquaux, G., Gramfort, A., et al. 2011, J. Mach. Learn. Res., 12,2825

Razzaque, S. 2013, PhRvD, 88, 081302

Reines, F. 1960, ARNPS, 10, 1

Reines, F., \& Cowan, C. L. 1956, Natur, 178, 446

Roberts, A. 1992, RvMP, 64, 259

Roulet, E., Sigl, G., van Vliet, A., \& Mollerach, S. 2013, JCAP, 01, 028

Schoenen, S., \& Raedel, L. 2015, ATel, 7856, 1

Schukraft, A. 2013, PhD thesis, RWTH Aachen Univ.

Senno, N., Murase, K., \& Meszaros, P. 2016, PhRvD, 93, 083003

Stecker, F. W. 2013, PhRvD, 88, 047301

Tamborra, I., Ando, S., \& Murase, K. 2014, JCAP, 1409, 043

Wakely, S. P., \& Horan, D. 2007, Proc. 30th ICRC, 3, 1341

Waxman, E. 2013, in Proc. 9th Rencontres du Vietnam: Windows on the Universe, ed. J. Dumarchez et al. (Quy Nhon: ICISE), 161

Wilks, S. S. 1938, Ann. Math. Statist., 9, 60 